\title{
Emergence of a singularity for Toeplitz determinants and Painlevé V
}

\author{
T. Claeys, A. Its, I. Krasovsky
}

June 7, 2018

\begin{abstract}
We obtain asymptotic expansions for Toeplitz determinants corresponding to a family of symbols depending on a parameter $t$. For $t$ positive, the symbols are regular so that the determinants obey Szegö's strong limit theorem. If $t=0$, the symbol possesses a Fisher-Hartwig singularity. Letting $t \rightarrow 0$ we analyze the emergence of a Fisher-Hartwig singularity and a transition between the two different types of asymptotic behavior for Toeplitz determinants. This transition is described by a special Painlevé V transcendent. A particular case of our result complements the classical description of Wu, McCoy, Tracy, and Barouch of the behavior of a 2-spin correlation function for a large distance between spins in the two-dimensional Ising model as the phase transition occurs.
\end{abstract}

\section{Introduction}

Consider the Toeplitz determinant with symbol $f(z) \in L^{1}(C)$, where $C$ is the unit circle:

$$
D_{n}=\operatorname{det}\left(f_{j-k}\right)_{j, k=0}^{n-1}, \quad f_{j}=\frac{1}{2 \pi} \int_{0}^{2 \pi} f\left(e^{i \theta}\right) e^{-i j \theta} d \theta .
$$

We are interested in the behavior of $D_{n}$ as $n \rightarrow \infty$.

If $\ln f(z)$ is sufficiently smooth on the unit circle (in particular, $f(z)$ is never zero for $z \in C$ and has no winding around the origin) so that $\ln f(z) \in L^{1}(C)$ and the sum

$$
\sum_{k=-\infty}^{\infty}|k|\left|(\ln f)_{k}\right|^{2}, \quad(\ln f)_{k}=\frac{1}{2 \pi} \int_{0}^{2 \pi} \ln f\left(e^{i \theta}\right) e^{-i k \theta} d \theta
$$

converges, then the asymptotics of $D_{n}$ are given by the strong Szegö limit theorem [22, 21, 25]:

$$
\ln D_{n}=\frac{n}{2 \pi} \int_{0}^{2 \pi} \ln f\left(e^{i \theta}\right) d \theta+\sum_{k=1}^{\infty} k(\ln f)_{k}(\ln f)_{-k}+o(1), \quad \text { as } n \rightarrow \infty .
$$

However, one often encounters a situation where the symbol possesses so-called Fisher-Hartwig singularities. In the case of only one such singularity, located at $z=1$, the symbol has the form:

$$
f(z)=|z-1|^{2 \alpha} z^{\beta} e^{-i \pi \beta} e^{V(z)}=(2-2 \cos \theta)^{\alpha} e^{i \beta(\theta-\pi)} e^{V\left(e^{i \theta}\right)}, \quad \text { for } 0<\theta<2 \pi,
$$


where $V(z)$ is a sufficiently smooth function (see [11) on the unit circle. The singularity at $z=1$ combines a jump-type (for $\alpha=0, \beta \neq 0$ ) and a root-type singularity (for $\beta=0$, $\alpha \neq 0$ ). For this symbol the sum (1.2) diverges, and therefore Szegö's theorem does not hold. The asymptotics for the Toeplitz determinant are given instead by the expression [8, 35, 6, 15, 11]:

$$
\begin{aligned}
& \ln D_{n}=n V_{0}+\sum_{k=1}^{\infty} k V_{k} V_{-k}-(\alpha-\beta) \sum_{k=1}^{\infty} V_{k}-(\alpha+\beta) \sum_{k=1}^{\infty} V_{-k} \\
& \quad+\left(\alpha^{2}-\beta^{2}\right) \ln n+\ln \frac{G(1+\alpha+\beta) G(1+\alpha-\beta)}{G(1+2 \alpha)}+o(1), \quad \text { as } n \rightarrow \infty
\end{aligned}
$$

if

$$
\alpha \pm \beta \neq-1,-2, \ldots
$$

with

$$
V_{k}=\frac{1}{2 \pi} \int_{0}^{2 \pi} V\left(e^{i \theta}\right) e^{-i k \theta} d \theta
$$

Here $G$ is Barnes' $G$-function, which is an entire function having the properties: $G(z+$ $1)=\Gamma(z) G(z)$, where $\Gamma(z)$ is Euler's $\Gamma$-function, and $G(1)=1, G(-k)=0$ for $k=$ $0,1,2, \ldots$ Note that if $V(z) \equiv 0$, there exists an explicit expression for $D_{n}$ with symbol $|z-1|^{2 \alpha} z^{\beta} e^{-i \pi \beta}$ in terms of $G$-functions [9, 10].

Suppose now that a symbol depends on a parameter $t(f(z)=f(z ; t))$ so that when $t>0$ the symbol is "regular", i.e. Szego"'s theorem holds for $D_{n}(t)$, while at $t=0$ the symbol has the form (1.4). The purpose of the present paper is to study the transition from (1.3) to (1.5) as $t \rightarrow 0$. Namely, consider the following symbol

$$
f(z)=\left(z-e^{t}\right)^{\alpha+\beta}\left(z-e^{-t}\right)^{\alpha-\beta} z^{-\alpha+\beta} e^{-i \pi(\alpha+\beta)} e^{V(z)}, \quad \alpha \pm \beta \neq-1,-2, \ldots,
$$

where $t \geq 0$ is sufficiently small and $\alpha, \beta \in \mathbb{C}$ with $\operatorname{Re} \alpha>-\frac{1}{2}$. We further assume that $V(z)$ is analytic in an annulus containing the unit circle and write it there in terms of its Fourier series

$$
V(z)=\sum_{k=-\infty}^{+\infty} V_{k} z^{k} .
$$

We define the powers in (1.7) with arguments between 0 and $2 \pi$. With this choice of branch cuts, $f$, and moreover $\ln f$, is analytic in $\mathbb{C} \backslash\left(\left[0, e^{-t}\right] \cup\left[e^{t},+\infty\right)\right)$ and, in particular, on the unit circle for $t>0$. Therefore, for any fixed $t>0$, the asymptotics of the Toeplitz determinant $D_{n}(t)$ are given by (1.3). Calculating the Fourier coefficients $(\ln f)_{k}$, we obtain

$$
\begin{aligned}
& \ln D_{n}(t)=n t(\alpha+\beta)+n V_{0} \\
& +\sum_{k=1}^{\infty} k\left[V_{k}-(\alpha+\beta) \frac{e^{-t k}}{k}\right]\left[V_{-k}-(\alpha-\beta) \frac{e^{-t k}}{k}\right]+o(1), \quad \text { as } n \rightarrow \infty, t>0 .
\end{aligned}
$$

For $t=0$, the symbol reduces to (1.4) (with analytic $V$ ). Therefore, for $t=0$, the asymptotics of $D_{n}(t)$ are given by (1.5). In the present paper we describe the transition from (1.9) to (1.5) when $t$ decreases to 0 . 
The paper also sets the stage for analysis of various other transition asymptotics for Toeplitz determinants, such as 2 singularities approaching each other; emergence of an arc of the unit circle where the symbol $f=0$ from 2 jump-type singularities at the ends of the arc, etc.

Our analysis explains to some extent the question of connection between Painlevé tau-functions and Toeplitz determinants which was noticed before: see [7] for a discussion and references. Historically and as the most prominent example, this connection appeared in the study of 2-spin correlation functions in the 2-dimensional Ising model. We discuss this in the following section.

\subsection{Application: the two-dimensional Ising model}

Transitions between Szegö weights and Fisher-Hartwig weights arise for example in the theory of solvable two-dimensional statistical models and one-dimensional Heisenberg spin chains. Recall the two-dimensional Ising model solved by Onsager (see, e.g., [32, 29]). In this model a $2 \mathcal{M} \times 2 \mathcal{N}$ rectangular lattice is considered with an associated spin variable $\sigma_{j k}$ taking the values 1 and -1 at each vertex $(j, k),-\mathcal{M} \leq j \leq \mathcal{M}-1$, $-\mathcal{N} \leq k \leq \mathcal{N}-1$. There are $2^{4 \mathcal{M N}}$ possible spin configurations $\{\sigma\}$ of the lattice (a configuration corresponds to values of all $\sigma_{j k}$ fixed). We associate with each configuration the energy of the nearest-neighbor coupling (imposing the cyclic boundary conditions on the lattice)

$$
E(\{\sigma\})=-\sum_{j=-\mathcal{M}}^{\mathcal{M}-1} \sum_{k=-\mathcal{N}}^{\mathcal{N}-1}\left(\gamma_{1} \sigma_{j k} \sigma_{j k+1}+\gamma_{2} \sigma_{j k} \sigma_{j+1 k}\right), \quad \gamma_{1}, \gamma_{2}>0
$$

The partition function at a temperature $T>0$ is equal to

$$
Z(T)=\sum_{\{\sigma\}} e^{-E(\{\sigma\}) / T}
$$

where the sum is over all configurations. A remarkable feature of this model is the presence of a thermodynamic phase transition in the limit of the infinite lattice at a certain temperature $T_{c}$ depending on $\gamma_{1}, \gamma_{2}$.

Define a 2-spin correlation function by the expression

$$
<\sigma_{00} \sigma_{n n}>=\lim _{\mathcal{M}, \mathcal{N} \rightarrow \infty} \frac{1}{Z(T)} \sum_{\{\sigma\}} \sigma_{00} \sigma_{n n} e^{-E(\{\sigma\}) / T} .
$$

For large $n$, this function measures the long-range order in the lattice at a temperature $T$, which determines magnetization. Indeed one can show that the spontaneous magnetization $M$ is given by the expression

$$
M=\sqrt{\lim _{n \rightarrow \infty}<\sigma_{00} \sigma_{n n}>} .
$$

It is a remarkable fact that the 2-spin correlation function is a Toeplitz determinant

$$
<\sigma_{00} \sigma_{n n}>=e^{n t / 2} D_{n}(t), \quad f(z ; t)=\left(z-e^{t}\right)^{-1 / 2}\left(z-e^{-t}\right)^{1 / 2} z^{-1 / 2} e^{i \pi / 2},
$$

where

$$
e^{t}=\sinh \frac{2 \gamma_{1}}{T} \sinh \frac{2 \gamma_{2}}{T},
$$


and the branches of the roots are chosen with the arguments from 0 to $2 \pi$. The symbol in (1.14) has the form (1.7) with

$$
\alpha=0, \quad \beta=-\frac{1}{2}, \quad V(z) \equiv 0 .
$$

The critical temperature $T_{c}$ is defined by the condition that $t=0$.

For $T<T_{c}$ we have $t>0$. Therefore the strong Szegő limit theorem (1.9) holds, and using the elementary identity

$$
\sum_{k=1}^{\infty} e^{-2 k t} / k=-\ln \left(1-e^{-2 t}\right),
$$

we rederive the well-known result

$$
\begin{aligned}
& e^{n t / 2} D_{n}(t)=\left(1-e^{-2 t}\right)^{1 / 4}(1+o(1))= \\
& {\left[1-\left(\sinh \frac{2 \gamma_{1}}{T} \sinh \frac{2 \gamma_{2}}{T}\right)^{-2}\right]^{1 / 4}(1+o(1)), \quad \text { as } n \rightarrow \infty, T<T_{c} .}
\end{aligned}
$$

The correlations tend to a constant as $n \rightarrow \infty$ : the model exhibits the long-range order. Notice that by (1.13), the asymptotics (1.18) imply the existence of the spontaneous magnetization for $T<T_{c}$ and the famous power-law decay of the magnetization $M \sim$ Const $\cdot\left(T_{c}-T\right)^{1 / 8}$ as $T \nearrow T_{c}$.

At $T=T_{c}$, we have $t=0$, and therefore a Fisher-Hartwig singularity with the parameters $\alpha=0, \beta=-1 / 2$ appears at $z=1$. In this case, (1.5) holds and we obtain (cf. [32, 29])

$$
D_{n}(0)=\frac{\sqrt{\pi} G(1 / 2)^{2}}{n^{1 / 4}}(1+o(1))
$$

so the correlations decrease as $n^{-1 / 4}, n \rightarrow \infty$ : the long-range order is destroyed. Note that as $V(z) \equiv 0$, there is an explicit expression for $D_{n}(0)$ (cf. the remark following $(1.5[1.6)$ ):

$$
D_{n}(0)=\left(\frac{2}{n}\right)^{n} \prod_{k=1}^{n-1}\left(1-\frac{1}{4 k^{2}}\right)^{k-n} .
$$

For $T>T_{c}$ we have $t<0$. The symbol still has a singularity at $z=1$ but now with the parameters $\alpha=0, \beta=-1$. This is the situation of a degenerate type of a Fisher-Hartwig singularity, and (1.5) does not hold in this case as one of the $G$-functions vanishes. Calculations [32] show an $n^{-1 / 2} e^{n t}$ decay of the correlations (1.12) as $n \rightarrow \infty$. There is no long-range order and $M=0$.

The transition $T \rightarrow T_{c}$ for large $n$ was studied in [36, 30, 34] (for a more general correlation function $\left\langle\sigma_{00} \sigma_{n m}>\right)$. The authors took $n \rightarrow \infty$ with $x=n\left(e^{2 t}-1\right)$ fixed and found, in particular, that in this limit

$$
n^{1 / 4}<\sigma_{00} \sigma_{n n}>\rightarrow F(x),
$$

where $F(x)$ is given in terms of a solution to Painlevé III equation (reducible to Painlevé $\mathrm{V}$ : see equation (1.28) below). Moreover, in [30], McCoy, Tracy and Wu evaluated the connection formulae for this Painlevé III function and showed that the limiting 
behavior of $F(x)$ as $x \rightarrow \infty$ and as $x \rightarrow 0$ formally matches the asymptotics (1.18) and (1.19), respectively. The matching with (1.19) was, however, checked only up to a multiplicative constant. A more detailed evaluation of the small $x$ behavior of the function $F(x)$ was carried out later by Tracy in [34, and reproduced exactly the constant in the critical point asymptotics (1.19). The calculations of [30] and 34] were based on an alternative representation of the function $F(x)$ as an infinite series of integrals and were rather involved.

The results of the present paper, namely a particular case of Theorem 1.4 below, fully describe (up to a possibility of a finite number of poles: see below) the transition of the correlation function (1.12) for large $n$ from $T<T_{c}$ to $T=T_{c}$, that is the transition from (1.18) to (1.19). Note that we do not rely on (1.20) as the parameter $x$ in our analysis is not necessarily fixed, in fact, our asymptotics for the correlation function are uniform in the whole range $x \in[0, \infty)$ (away from a finite number of positive points). More precisely, our asymptotics as $n \rightarrow \infty$ are uniform for all $T \in\left[T_{1}, T_{c}\right]$ (away from a finite number of positive $x$ 's) for some $T_{1}<T_{c}$ with $T_{c}-T_{1}$ sufficiently small.

The description of the Ising double scaling theory which we obtain as a particular case of Theorem 1.4 is in agreement with the classical results of [36, 30, 34] (see also Remark 1.7 below). Furthermore, by obtaining the uniform asymptotics for the whole transition range of temperatures $T \leq T_{c}$, we complement the analysis of this case of the Wu-McCoy-Tracy-Barouch scaling theory.

Another example where Theorems 1.1, 1.4 below can be applied is the so-called emptiness formation probability in a Heisenberg spin chain [20].

\section{$1.2 \quad$ Statement of results}

Consider the second order ODE

$$
\begin{aligned}
\left(x \frac{d^{2} \sigma}{d x^{2}}\right)^{2}=\left(\sigma-x \frac{d \sigma}{d x}+2(\right. & \left.\left.\frac{d \sigma}{d x}\right)^{2}+2 \alpha \frac{d \sigma}{d x}\right)^{2} \\
& -4\left(\frac{d \sigma}{d x}\right)^{2}\left(\frac{d \sigma}{d x}+\alpha+\beta\right)\left(\frac{d \sigma}{d x}+\alpha-\beta\right) .
\end{aligned}
$$

This is the Jimbo-Miwa-Okamoto $\sigma$-form [25, 26] of the fifth Painlevé equation

$$
u_{x x}=\left(\frac{1}{2 u}+\frac{1}{u-1}\right) u_{x}^{2}-\frac{1}{x} u_{x}+\frac{(u-1)^{2}}{x^{2}}\left(A u+\frac{B}{u}\right)+\frac{C u}{x}+D \frac{u(u+1)}{u-1},
$$

with the parameters $A, B, C, D$ given by

$$
A=\frac{1}{2}(\alpha-\beta)^{2}, \quad B=-\frac{1}{2}(\alpha+\beta)^{2}, \quad C=1+2 \beta, \quad D=-\frac{1}{2} .
$$

In the following theorem, we give the asymptotic expansion for the Toeplitz determinant with symbol (1.7) as $n \rightarrow \infty$ which is valid uniformly for $0<t<t_{0}$. Our asymptotic expansion interpolates between Szegö and Fisher-Hartwig asymptotics.

Theorem 1.1 Let $\alpha \in \mathbb{R}, \alpha>-\frac{1}{2}, \beta \in i \mathbb{R}$. Let $f$ be defined by (1.7) and consider the Toeplitz determinant $D_{n}(t)$ defined by (1.1) corresponding to this symbol. The following 
asymptotic expansion holds as $n \rightarrow \infty$ with the error term o(1) uniform for $0<t<t_{0}$ where $t_{0}$ is sufficiently small:

$$
\begin{array}{r}
\ln D_{n}(t)=n V_{0}+(\alpha+\beta) n t+\sum_{k=1}^{\infty} k\left[V_{k}-(\alpha+\beta) \frac{e^{-t k}}{k}\right]\left[V_{-k}-(\alpha-\beta) \frac{e^{-t k}}{k}\right] \\
+\ln \frac{G(1+\alpha+\beta) G(1+\alpha-\beta)}{G(1+2 \alpha)}+\Omega(2 n t)+o(1),
\end{array}
$$

where $G(z)$ is Barnes' G-function, and

$$
\Omega(2 n t)=\int_{0}^{2 n t} \frac{\sigma(x)-\alpha^{2}+\beta^{2}}{x} d x+\left(\alpha^{2}-\beta^{2}\right) \ln 2 n t .
$$

The function $\sigma(x)$ is a particular solution to the equation (1.21) which is real analytic on $(0,+\infty)$, and has the following asymptotics for $x>0$ :

$$
\sigma(x)= \begin{cases}\alpha^{2}-\beta^{2}+\frac{\alpha^{2}-\beta^{2}}{2 \alpha}\left\{x-x^{1+2 \alpha} C(\alpha, \beta)\right\}(1+\mathcal{O}(x)), & x \rightarrow 0, \quad 2 \alpha \notin \mathbb{Z} \\ \alpha^{2}-\beta^{2}+\mathcal{O}(x)+\mathcal{O}\left(x^{1+2 \alpha}\right)+\mathcal{O}\left(x^{1+2 \alpha} \ln x\right), & x \rightarrow 0, \quad 2 \alpha \in \mathbb{Z} \\ x^{-1+2 \alpha} e^{-x} \frac{-1}{\Gamma(\alpha-\beta) \Gamma(\alpha+\beta)}\left(1+\mathcal{O}\left(\frac{1}{x}\right)\right), & x \rightarrow+\infty,\end{cases}
$$

with

$$
C(\alpha, \beta)=\frac{\Gamma(1+\alpha+\beta) \Gamma(1+\alpha-\beta)}{\Gamma(1-\alpha+\beta) \Gamma(1-\alpha-\beta)} \frac{\Gamma(1-2 \alpha)}{\Gamma(1+2 \alpha)^{2}} \frac{1}{1+2 \alpha},
$$

where $\Gamma(z)$ is Euler's $\Gamma$-function.

Remark 1.2 Later on, we will construct $\sigma(x)$ explicitly in terms of a Riemann-Hilbert problem.

Remark 1.3 With increasing effort, one can calculate more terms in the expansion (1.24) using our approach.

The function $\sigma=\sigma(x ; \alpha, \beta)$ is defined for $x \in \mathbb{C}$ with a cut from zero to infinity. It is analytic in the cut plane apart from possible poles. Asymptotics (1.26) imply that there are no poles for $x$ positive and sufficiently large. Hence the number of possible poles of $\sigma(x)$ on $(0,+\infty)$ is finite. We show below that for $\alpha>-\frac{1}{2}$ real, $\beta$ imaginary, there are no poles on the real half-axis $(0,+\infty)$. Therefore we took the intervals of the real line as a path of integration in (1.25). For $\beta$ arbitrary, $\operatorname{Re} \alpha>-1 / 2$, a similar result holds, but we have to choose a path of integration in the complex plane avoiding possible poles which we denote $\left\{x_{1}, \ldots, x_{\ell}\right\}$. Namely, we have

Theorem 1.4 Let $\alpha, \beta \in \mathbb{C}$ with $\operatorname{Re} \alpha>-\frac{1}{2}, \alpha \pm \beta \neq-1,-2, \ldots$, and let $s_{\delta}$ denote a sector $-\pi / 2+\delta<\arg x<\pi / 2-\delta, 0<\delta<\pi / 2$. Let $f$ be defined by (1.7) and consider the Toeplitz determinants $D_{n}(t)$ defined by (1.1) corresponding to this symbol. There exists a finite set $\left\{x_{1}, \ldots, x_{\ell}\right\} \in s_{\delta}$ (with $\ell=\ell(\alpha, \beta, \delta)$ and $x_{j}=x_{j}(\alpha, \beta) \neq 0$ ) such that the expansion (1.24) holds uniformly for $t \in s_{\delta},|t|<t_{0}$ (with $t_{0}$ sufficiently small) as long as 2 nt remains bounded away from the set $\left\{x_{1}, \ldots, x_{\ell}\right\}$. The function $\Omega$ is defined by (1.25), where the path of integration is chosen in $s_{\delta}$, connecting 0 with $2 n t$ and not containing any of the points $\left\{x_{1}, \ldots, x_{\ell}\right\}$. Moreover $\sigma(x)$ solves the ODE (1.21) and has the asymptotics in the mentioned sector given by (1.26). 
Remark 1.5 It follows from the representation (1.24) that the residue of $\frac{1}{x} \sigma(x)$ at each of its poles in the sector $-\pi / 2<\arg x<\pi / 2$ is an entire number. Different choices of the integration contour in (1.25) correspond, in general, to different branches of $\ln D_{n}(t)$. If $\alpha$ and $\beta$ are such that $\frac{1}{x} \sigma(x)$ has a pole $x$, the determinant $D_{n}(t)$ is zero at $2 n t=x($ up to an $\mathcal{O}(1 / n)$ error term).

Remark 1.6 From now on, we will always consider $t \geq 0$ for simplicity. The extension to $t \in s_{\delta}$ is straightforward.

Remark 1.7 In the example of the Ising model discussed in the previous section, we have $\alpha=0, \beta=-1 / 2$ (see 1.16), and equation (1.21) becomes

$$
\left(x \frac{d^{2} \sigma}{d x^{2}}\right)^{2}=\left(\sigma-x \frac{d \sigma}{d x}+2\left(\frac{d \sigma}{d x}\right)^{2}\right)^{2}-4\left(\frac{d \sigma}{d x}\right)^{2}\left(\left(\frac{d \sigma}{d x}\right)^{2}-\frac{1}{4}\right) .
$$

This is exactly the equation which was obtained in [26] for the function

$$
\zeta(x) \equiv x \frac{d}{d x} \ln F(x)-\frac{1}{4},
$$

where $F(x)$ is the right hand side of the Ising double scaling limit (1.20) (see also equation (4.16), with $r=x / 2$, in [29]). It follows immediately from our main result (1.24) that the function $\zeta(x)$ in [26] and our function $\sigma(x)$ coincide:

$$
\zeta(x)=\sigma(x) .
$$

Thus the application of our Theorem 1.4 to the Toeplitz determinant (1.14) yields the complete analysis (up to the question of existence of a finite number of positive poles $x_{j}$ ) for $T \leq T_{c}$ of the Jimbo-Miwa Painlevé V version of the Wu-McCoy-Tracy-Barouch scaling theory for the 2D Ising model.

From the expansion (1.24), we can recover the Fisher-Hartwig asymptotics for $\ln D_{n}(0)$. Let $t \rightarrow 0$, and $n$ fixed in (1.24). Then, using (1.25) and (1.26), we obtain that $\Omega(2 n t)=\left(\alpha^{2}-\beta^{2}\right) \ln (2 n t)+o(1)$ if $\operatorname{Re} \alpha>-\frac{1}{2}$. Substituting this into (1.24) and recalling (1.17), we obtain (1.5).

The expansion (1.24) should also be consistent with the Szegö asymptotics for $t$ fixed. We see immediately that the $\mathcal{O}(n)$ term gives, for a fixed $t$, the corresponding term in the Szegö asymptotics. Consistency of the $\mathcal{O}(1)$ terms, however, yields an interesting identity involving the Painlevé function $\sigma(x)$ via (1.25):

$$
\Omega(+\infty)=-\ln \frac{G(1+\alpha+\beta) G(1+\alpha-\beta)}{G(1+2 \alpha)} .
$$

\subsection{The Painlevé V Riemann-Hilbert problem}

We can say more about the function $\sigma(x)$ than we did in Theorem 1.1; we can construct it explicitly in terms of a Riemann-Hilbert $(\mathrm{RH})$ problem. Consider the contour $\Gamma=$ $\cup_{j=1}^{6} \Gamma_{j}$ in the complex plane (see Figure 1), with

$$
\begin{array}{lll}
\Gamma_{1}=\frac{1}{2}+e^{i \frac{\pi}{4}} \mathbb{R}^{+}, & \Gamma_{2}=\frac{1}{2}+e^{i \frac{3 \pi}{4}} \mathbb{R}^{+}, & \Gamma_{3}=\frac{1}{2}+e^{i \frac{5 \pi}{4}} \mathbb{R}^{+}, \\
\Gamma_{4}=\frac{1}{2}+e^{i \frac{7 \pi}{4}} \mathbb{R}^{+}, & \Gamma_{5}=(1,+\infty), & \Gamma_{6}=(0,1),
\end{array}
$$




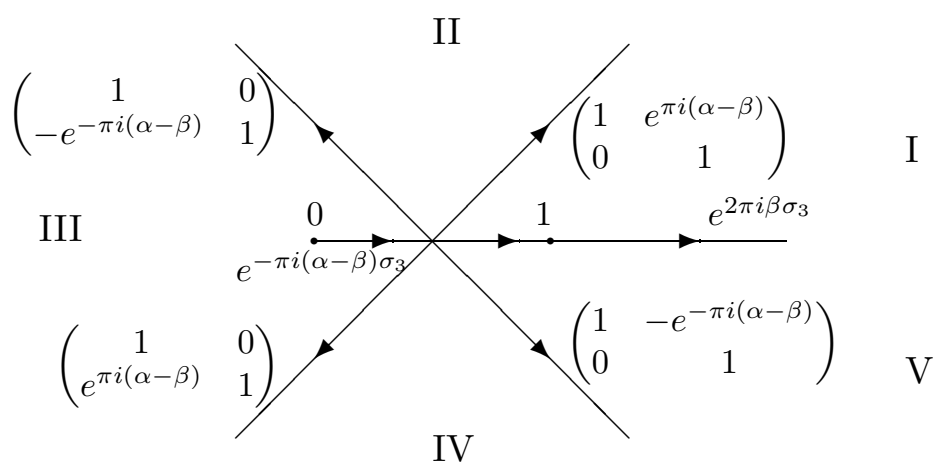

Figure 1: The jump contour and jump matrices for $\Psi$.

with $\Gamma_{1}, \ldots, \Gamma_{5}$ oriented towards infinity and $\Gamma_{6}$ oriented to the right. By a standard convention, the "+" side of the curve is on the left as one faces the direction of the curve's orientation.

Let $\operatorname{Re} \alpha>-\frac{1}{2}$ and consider the following $\mathrm{RH}$ problem for $\Psi=\Psi(\zeta ; x, \alpha, \beta)$.

\section{RH problem for $\Psi$}

(a) $\Psi: \mathbb{C} \backslash \Gamma \rightarrow \mathbb{C}^{2 \times 2}$ is analytic.

(b) $\Psi$ has continuous boundary values on $\Gamma \backslash\left\{0, \frac{1}{2}, 1\right\}$, and they are related as follows,

$$
\begin{array}{ll}
\Psi_{+}(\zeta)=\Psi_{-}(\zeta)\left(\begin{array}{cc}
1 & e^{\pi i(\alpha-\beta)} \\
0 & 1
\end{array}\right), & \text { for } \zeta \in \Gamma_{1}, \\
\Psi_{+}(\zeta)=\Psi_{-}(\zeta)\left(\begin{array}{cc}
1 & 0 \\
-e^{-\pi i(\alpha-\beta)} & 1
\end{array}\right), & \text { for } \zeta \in \Gamma_{2}, \\
\Psi_{+}(\zeta)=\Psi_{-}(\zeta)\left(\begin{array}{cc}
1 & 0 \\
e^{\pi i(\alpha-\beta)} & 1
\end{array}\right), & \text { for } \zeta \in \Gamma_{3}, \\
\Psi_{+}(\zeta)=\Psi_{-}(\zeta)\left(\begin{array}{cc}
1 & -e^{-\pi i(\alpha-\beta)} \\
0 & 1
\end{array}\right), & \text { for } \zeta \in \Gamma_{4}, \\
\Psi_{+}(\zeta)=\Psi_{-}(\zeta) e^{2 \pi i \beta \sigma_{3}}, & \text { for } \zeta \in \Gamma_{5}, \\
\Psi_{+}(\zeta)=\Psi_{-}(\zeta) e^{-\pi i(\alpha-\beta) \sigma_{3}}, & \text { for } \zeta \in \Gamma_{6},
\end{array}
$$

with $\sigma_{3}=\left(\begin{array}{cc}1 & 0 \\ 0 & -1\end{array}\right)$.

(c) $\Psi$ has the following behavior as $\zeta \rightarrow \infty$ (for some matrices $C_{1}=C_{1}(x, \alpha, \beta)$, $\left.C_{2}=C_{2}(x, \alpha, \beta)\right)$

$$
\Psi(\zeta)=\left(I+\frac{C_{1}}{\zeta}+\frac{C_{2}}{\zeta^{2}}+\mathcal{O}\left(\zeta^{-3}\right)\right) \zeta^{-\beta \sigma_{3}} e^{-\frac{x}{2} \zeta \sigma_{3}}
$$

$(\mathrm{d} 0)$ As $\zeta \rightarrow 0$

$$
\Psi(\zeta)=\mathcal{O}\left(\begin{array}{ll}
|\zeta|^{\frac{\alpha-\beta}{2}} & |\zeta|^{-\frac{\alpha-\beta}{2}} \\
|\zeta|^{\frac{\alpha-\beta}{2}} & |\zeta|^{-\frac{\alpha-\beta}{2}}
\end{array}\right) .
$$


(d1) As $\zeta \rightarrow 1$,

$$
\Psi(\zeta)=\mathcal{O}\left(\begin{array}{ll}
|\zeta-1|^{-\frac{\alpha+\beta}{2}} & |\zeta-1|^{\frac{\alpha+\beta}{2}} \\
|\zeta-1|^{-\frac{\alpha+\beta}{2}} & |\zeta-1|^{\frac{\alpha+\beta}{2}}
\end{array}\right) .
$$

Furthermore $\Psi$ is bounded near $\frac{1}{2}$.

The RH conditions imply (by a standard argument) that the determinant of the solution $\Psi$ (which is, if it exists, unique) is identically equal to 1 , and consequently we have using (1.38) that $\operatorname{tr} C_{1}=0$. Let us denote the matrix elements of $C_{1}$ by

$$
C_{1}(x)=\left(\begin{array}{cc}
q(x) & r(x) \\
t(x) & -q(x)
\end{array}\right)
$$

Define the functions $v$ and $u$ in terms of the matrix elements of $C_{1}$ :

$$
\begin{aligned}
& v(x)=\frac{\alpha+\beta}{2}-q(x)-x r(x) t(x), \\
& u(x)=1+\frac{x t}{(2 \beta+1-x) t(x)+x t^{\prime}(x)} .
\end{aligned}
$$

We will show in Section 4.3 below that

$$
\sigma(x)=\int_{x}^{+\infty} v(\xi) d \xi
$$

is the function appearing in Theorem 1.1 and Theorem 1.4. The RH problem for $\Psi$ is a special case of the $\mathrm{RH}$ problem associated to the fifth Painlevé equation, see e.g. [16, 18.

We prove the following.

Theorem 1.8 Let $\alpha, \beta \in \mathbb{C}$ and $\operatorname{Re} \alpha>-\frac{1}{2}$.

(i) The RH problem for $\Psi$ is uniquely solvable for all $x>0$ except possibly for a finite number of positive $x$-values. We denote the $x$-values for which the RH problem is not solvable by $\left\{x_{1}, \ldots, x_{k}\right\}$, with $x_{j}=x_{j}(\alpha, \beta)$ and $k=k(\alpha, \beta)$.

(ii) If $\operatorname{Im} \alpha=0$ and $\operatorname{Re} \beta=0$ the $R H$ problem is solvable for all positive $x$-values.

(iii) The function $v$ defined by (1.41) is analytic in $(0,+\infty) \backslash\left\{x_{1}, \ldots, x_{k}\right\}$, and solves, together with $u$ defined by (1.42), the system

$$
\begin{aligned}
& x u_{x}=x u-2 v(u-1)^{2}+(u-1)[(\alpha-\beta) u-\beta-\alpha], \\
& x v_{x}=u v[v-\alpha+\beta]-\frac{v}{u}(v-\beta-\alpha) .
\end{aligned}
$$

(iv) The function $v$ has the asymptotics given by

$$
v(x)= \begin{cases}-\frac{\alpha^{2}-\beta^{2}}{2 \alpha}\left\{1-(2 \alpha+1) x^{2 \alpha} C(\alpha, \beta)\right\}(1+\mathcal{O}(x)), & x \rightarrow 0, \quad 2 \alpha \notin \mathbb{Z}, \\ \mathcal{O}(1)+\mathcal{O}\left(x^{2 \alpha}\right)+\mathcal{O}\left(x^{2 \alpha} \ln x\right), & x \rightarrow 0, \quad 2 \alpha \in \mathbb{Z} \\ x^{-1+2 \alpha} e^{-x} \frac{-1}{\Gamma(\alpha-\beta) \Gamma(\alpha+\beta)}\left(1+\mathcal{O}\left(\frac{1}{x}\right)\right), & x \rightarrow+\infty,\end{cases}
$$

where $C(\alpha, \beta)$ is defined in 1.27). 
In addition, we have

$$
\int_{0}^{+\infty} v(x) d x=\alpha^{2}-\beta^{2}
$$

if $\operatorname{Im} \alpha=0$ and $\operatorname{Re} \beta=0$. In the general case, $\alpha, \beta \in \mathbb{C}, \operatorname{Re} \alpha>-\frac{1}{2}$, equation (1.47) holds up to addition of $2 \pi i m, m \in \mathbb{Z}$, with the path of integration avoiding $\left\{x_{1}, \ldots, x_{k}\right\}$.

Part (iii) of the theorem follows from a standard Lax pair argument and was proved in [18, 16] for a slightly different but equivalent $\mathrm{RH}$ problem. That proof applies to our $\mathrm{RH}$ problem as well, and implies moreover that the $\mathrm{RH}$ solution is meromorphic in $x$ for $x \in \mathbb{C} \backslash\{0\}$. We will come back to this in Section 4 . We prove part (iv) by performing the Deift-Zhou steepest descent analysis for the RH problem. This asymptotic analysis also implies the solvability of the $\mathrm{RH}$ problem for large $x$ and small $x$, and by meromorphicity in $x$, this leads to the statement (i). We prove part (ii) by applying the technique of a vanishing lemma to the $\mathrm{RH}$ problem for $\Psi$.

Remark 1.9 The system (1.44)-(1.45) is related to the Painlevé V equation: eliminating $v$, we easily verify that $u$ solves the Painlevé $\mathrm{V}$ equation (1.22)-(1.23). Asymptotic expansions as $x \rightarrow 0$ and as $x \rightarrow \infty$ for various solutions to the fifth Painlevé equation and the system (1.44)-(1.45) were obtained in several works, see e.g. [1, 2, 3, 25, 31, 33. The solution $v$ which is of interest to us decays exponentially at $+\infty$, is integrable near 0 if $\operatorname{Re} \alpha>-\frac{1}{2}$, and it has no poles on $(0,+\infty)$ if $\alpha>-\frac{1}{2} \in \mathbb{R}$ and $\beta \in i \mathbb{R}$. Note that the asymptotics of $\sigma(x)$ (1.26) follow from (1.46) and (1.47) by (1.43). Although we obtained the connection formulae (1.26) as a by-product of our analysis, we would expect that these asymptotics can be found in the general list of connection formulae for the fifth Painlevé equation obtained in [1, 2, 3].

\section{Outline of the paper}

The proofs of Theorem 1.1 and Theorem 1.4 are based on a well-known connection between Toeplitz determinants and orthogonal polynomials on the unit circle. In Section 2. we obtain a differential identity for $\ln D_{n}(t)$ in terms of the polynomials orthogonal on the unit circle with weight $f(z)$. In Section 3, we obtain large $n$ asymptotics for these orthogonal polynomials from a RH problem. The asymptotics will be given in terms of a model RH problem which we study in detail in Section 4, where we also give a proof of Theorem 1.8. In Section 5, we use the previously obtained asymptotics for the orthogonal polynomials and the results of Section 4 to integrate the differential identity for $\ln D_{n}(t)$, which leads to Theorem 1.1 and Theorem 1.4.

Throughout the paper, we choose the branches of logarithms and roots corresponding to arguments between 0 and $2 \pi$, unless stated otherwise.

\section{RH problem for orthogonal polynomials and a differen- tial identity for the Toeplitz determinants}

Our analysis is based on a classical connection between Toeplitz determinants and orthogonal polynomials. Assume that for some $n>0 D_{n}, D_{n+1} \neq 0$, and define a 
polynomial $\phi_{n}(z)$ in terms of the Fourier coefficients of $f(z)$ as follows:

$$
\phi_{n}(z)=\frac{1}{\sqrt{D_{n} D_{n+1}}}\left|\begin{array}{cccc}
f_{0} & f_{-1} & \cdots & f_{-n} \\
f_{1} & f_{0} & \cdots & f_{-n+1} \\
\vdots & \vdots & & \vdots \\
f_{n-1} & f_{n-2} & \cdots & f_{-1} \\
1 & z & \cdots & z^{n}
\end{array}\right| .
$$

The leading coefficient of $\phi_{n}$ is then equal to

$$
\chi_{n}=\sqrt{\frac{D_{n}}{D_{n+1}}} .
$$

There holds the orthogonality relation

$$
\frac{1}{2 \pi} \int_{C} \phi_{n}(z) z^{-j} f(z) \frac{d z}{i z}=\chi_{n}^{-1} \delta_{j n}, \quad j=0,1, \ldots n
$$

where $C$ is the unit circle oriented in the counterclockwise direction. Similarly, let $\hat{\phi}_{n}(z)$ be defined by

$$
\hat{\phi}_{n}(z)=\frac{1}{\sqrt{D_{n} D_{n+1}}}\left|\begin{array}{ccccc}
f_{0} & f_{-1} & \cdots & f_{-n+1} & 1 \\
f_{1} & f_{0} & \cdots & f_{-n+2} & z \\
\vdots & \vdots & & \vdots & \\
f_{n} & f_{n-1} & \cdots & f_{1} & z^{n}
\end{array}\right| .
$$

Then $\hat{\phi}_{n}$ has the same leading coefficient $\chi_{n}$ as $\phi_{n}$, and

$$
\frac{1}{2 \pi} \int_{C} \hat{\phi}_{n}\left(z^{-1}\right) z^{j} f(z) \frac{d z}{i z}=\chi_{n}^{-1} \delta_{j n}, \quad j=0,1, \ldots, n .
$$

If $D_{n} \neq 0$ for $n=1, \ldots$ (and we set $\left.D_{0} \equiv 1, \phi_{0}(z)=\hat{\phi}_{0}(z)=1 / \sqrt{D_{1}}\right)$, the system of polynomials $\phi_{n}$ and $\hat{\phi}_{n}, n=0,1, \ldots$ exists and can be characterized by the orthonormality relations

$$
\frac{1}{2 \pi} \int_{C} \phi_{k}(z) \hat{\phi}_{m}\left(z^{-1}\right) f(z) \frac{d z}{i z}=\delta_{k m}, \quad k, m=0,1, \ldots
$$

If the symbol $f$ is positive on the unit circle $C$, it is a classical fact (which follows, e.g., from the representation of a Toeplitz determinant as a multiple integral) that $D_{n}(f)>0$ for all $n \geq 0$, and the system of orthogonal polynomials exists.

Assume that $D_{n-1}, D_{n}, D_{n+1} \neq 0, t>0$, and define the function $Y(z ; n)$ as follows

$$
Y(z)=\left(\begin{array}{cc}
\chi_{n}^{-1} \phi_{n}(z) & \chi_{n}^{-1} \int_{C} \frac{\phi_{n}(\xi)}{\xi-z} \frac{f(\xi) d \xi}{2 \pi i \xi n} \\
-\chi_{n-1} z^{n-1} \hat{\phi}_{n-1}\left(z^{-1}\right) & -\chi_{n-1} \int_{C} \frac{\hat{\phi}_{n-1}\left(\xi^{-1}\right)}{\xi-z} \frac{f(\xi) d \xi}{2 \pi i \xi}
\end{array}\right) .
$$

Then $Y$ is the unique solution of the following $\mathrm{RH}$ problem with a jump on the counterclockwise oriented unit circle $C$. 


\section{RH problem for $Y$}

(a) $Y: \mathbb{C} \backslash C \rightarrow \mathbb{C}^{2 \times 2}$ is analytic.

(b) $Y_{+}(z)=Y_{-}(z)\left(\begin{array}{cc}1 & z^{-n} f(z) \\ 0 & 1\end{array}\right), \quad$ for $z \in C$.

(c) $Y(z)=(I+\mathcal{O}(1 / z))\left(\begin{array}{cc}z^{n} & 0 \\ 0 & z^{-n}\end{array}\right), \quad$ as $z \rightarrow \infty$.

A general fact that orthogonal polynomials can be so represented as a solution of a RH problem was noticed in [17] (for polynomials on the line) and extended for polynomials on the circle in [4].

In the next section we will show that the $\mathrm{RH}$ problem for $Y(z ; n, t)$ is solvable (and therefore the orthogonal polynomials exist and the coefficients $\chi_{n}$ are nonzero) for all $n$ larger than some $n_{0}(\alpha, \beta)$ provided $2 n t$ is bounded away from a certain finite set of points (in particular, see Proposition 2.1 below, $D_{n} \neq 0$ ). The number $n_{0}(\alpha, \beta)$ is bounded for $\alpha$ and $\beta$ in a bounded set.

Our next aim is to express $\frac{d}{d t} \ln D_{n}(t)$ in terms of the entries of the RH solution $Y$. We prove the following.

Proposition 2.1 Let $t>0$ and $n \in \mathbb{N}$. Suppose that the $R H$ problem for $Y(z ; n, t)$ is solvable. Then $D_{n} \neq 0$, and the following differential identity holds:

$$
\frac{d}{d t} \ln D_{n}(t)=-(\alpha+\beta) e^{t}\left(Y^{-1} \frac{d Y}{d z}\right)_{22}\left(e^{t}\right)+(\alpha-\beta) e^{-t}\left(Y^{-1} \frac{d Y}{d z}\right)_{22}\left(e^{-t}\right) .
$$

Proof. We will follow the approach of [24]. Let us start with the expression

$$
D_{n}(f)=\operatorname{det}\left(I-K_{n}\right),
$$

where $K_{n}$ is an integral operator acting on $L^{2}(C)$ with kernel

$$
K_{n}\left(z, z^{\prime}\right)=\frac{\left(z / z^{\prime}\right)^{n}-1}{z-z^{\prime}} \frac{1-f\left(z^{\prime}\right)}{2 \pi i} .
$$

This fact is easy to verify by considering the matrix expression for $K_{n}$ in the basis $\left\{z^{k}\right\}$, $k=-\infty, \ldots, \infty$.

As follows from the theory of "integrable" Fredholm operators (see, e.g. [24]), the solvability of the $\mathrm{RH}$ problem for $Y(z ; n, t)$ implies that the operator $1-K_{n}$ is invertible. Therefore, $D_{n}(f)=\operatorname{det}\left(I-K_{n}\right) \neq 0$.

Consider

$$
\frac{d}{d t} \ln D_{n}(f)=\frac{d}{d t} \operatorname{tr} \ln \left(I-K_{n}\right)=-\operatorname{tr}\left(I-K_{n}\right)^{-1} \frac{d K_{n}}{d t} .
$$

Since

$$
\frac{d f}{d t}=\left(-\frac{\alpha+\beta}{z-e^{t}} e^{t}+\frac{\alpha-\beta}{z-e^{-t}} e^{-t}\right) f
$$

we have

$$
\frac{d K_{n}}{d t}=K_{n}^{(1)}-K_{n}^{(2)},
$$


where

$$
K_{n}^{(2)}\left(z, z^{\prime}\right)=\frac{\alpha-\beta}{z^{\prime}-e^{-t}} \frac{\left(z / z^{\prime}\right)^{n}-1}{z-z^{\prime}} \frac{f\left(z^{\prime}\right)}{2 \pi i} e^{-t}
$$

and

$$
K_{n}^{(1)}\left(z, z^{\prime}\right)=\Lambda_{1}\left(z, z^{\prime}\right)-\frac{\alpha+\beta}{z^{\prime}-e^{t}} \frac{\left(z / z^{\prime}\right)^{n}-1}{z-z^{\prime}} \frac{1-f\left(z^{\prime}\right)}{2 \pi i} e^{t} .
$$

Here

$$
\Lambda_{1}\left(z, z^{\prime}\right)=\frac{\alpha+\beta}{z^{\prime}-e^{t}} \frac{e^{t}}{2 \pi i} \frac{\left(z / z^{\prime}\right)^{n}-1}{z-z^{\prime}} .
$$

The reason to single out $\Lambda_{1}$ will soon become clear. By a residue calculation, we obtain

$$
\left(\Lambda_{1} K_{n}\right)\left(z, z^{\prime}\right)=(\alpha+\beta) e^{t} \frac{1-f\left(z^{\prime}\right)}{2 \pi i} \frac{1}{z-e^{t}}\left[\frac{\left(z / z^{\prime}\right)^{n}-1}{z-z^{\prime}}+\frac{1}{e^{t}-z^{\prime}}\left(\left(\frac{z}{e^{t}}\right)^{n}-\left(\frac{z}{z^{\prime}}\right)^{n}\right)\right] .
$$

We can now rewrite (2.15) as follows:

$$
K_{n}^{(1)}\left(z, z^{\prime}\right)=\left(\Lambda_{1}\left(I-K_{n}\right)\right)\left(z, z^{\prime}\right)+(\alpha+\beta) e^{t} \frac{1-f\left(z^{\prime}\right)}{2 \pi i} \frac{1-\left(z / e^{t}\right)^{n}}{\left(z-e^{t}\right)\left(z^{\prime}-e^{t}\right)} .
$$

Defining the following 2-component vectors

$$
\hat{f}(z)=\left(\begin{array}{c}
z^{n} \\
1
\end{array}\right), \quad \hat{g}(z)=\frac{1-f(z)}{2 \pi i}\left(\begin{array}{c}
z^{-n} \\
-1
\end{array}\right), \quad \widetilde{f}(z)=\frac{\hat{f}(z)}{z-e^{t}}, \quad \widetilde{g}(z)=\frac{\hat{g}(z)}{z-e^{t}},
$$

we can write (2.17) in the final form:

$$
K_{n}^{(1)}\left(z, z^{\prime}\right)=\left(\Lambda_{1}\left(I-K_{n}\right)\right)\left(z, z^{\prime}\right)+(\alpha+\beta) e^{t}\left(e^{-n t} \widetilde{f}_{1}(z) \widetilde{g}_{2}\left(z^{\prime}\right)-\widetilde{f}_{2}(z) \widetilde{g}_{2}\left(z^{\prime}\right)\right)
$$

On the other hand, let us define $F_{j}=\left(I-K_{n}\right)^{-1} \hat{f}_{j}$. Then

$$
\frac{F_{j}(z)}{z-e^{t}}-\int_{C} \frac{K_{n}\left(z, z^{\prime}\right)}{z-e^{t}} F_{j}\left(z^{\prime}\right) d z^{\prime}=\widetilde{f}_{j}(z), \quad j=1,2 .
$$

Noting that

$$
K_{n}\left(z, z^{\prime}\right)=\frac{\hat{f}(z)^{T} \hat{g}\left(z^{\prime}\right)}{z-z^{\prime}}
$$

we can write the above equation in the form

$$
\frac{F_{j}(z)}{z-e^{t}}-\int_{C} K_{n}\left(z, z^{\prime}\right) \frac{F_{j}\left(z^{\prime}\right)}{z^{\prime}-e^{t}} d z^{\prime}+\int_{C} \widetilde{f}(z)^{T} \hat{g}\left(z^{\prime}\right) \frac{F_{j}\left(z^{\prime}\right)}{z^{\prime}-e^{t}} d z^{\prime}=\widetilde{f}_{j}(z) .
$$

Applying $\left(I-K_{n}\right)^{-1}$ to both sides, we obtain

$$
\sum_{k=1}^{2} m_{j k}\left(e^{t}\right) \widetilde{F}_{k}(z)=\frac{1}{z-e^{t}} F_{j}(z), \quad j=1,2,
$$

where $\widetilde{F}_{k}=\left(I-K_{n}\right)^{-1} \widetilde{f}_{k}$ and

$$
m_{j k}\left(e^{t}\right)=\delta_{j k}-\int_{C} F_{j}(z) \hat{g}_{k}(z) \frac{d z}{z-e^{t}}, \quad j, k=1,2 .
$$


Thus we have for the 2-component vector $(\operatorname{det} m=1$, see [24])

$$
\widetilde{F}(z)=\frac{1}{z-e^{t}} m^{-1}\left(e^{t}\right) F(z)=\frac{1}{z-e^{t}}\left(\begin{array}{c}
m_{22} F_{1}-m_{12} F_{2} \\
-m_{21} F_{1}+m_{11} F_{2}
\end{array}\right) .
$$

As is shown in ([24], Eq. (2.16) up to a different notation), the matrix $m$ is related to $Y$. For $|z|>1$,

$$
Y(z)=\left(\begin{array}{cc}
m_{11} z^{n}+m_{12} & -m_{12} z^{-n} \\
-m_{21} z^{n}-m_{22} & m_{22} z^{-n}
\end{array}\right)
$$

Using the definition $\widetilde{F}=\left(I-K_{n}\right)^{-1} \widetilde{f}$, equations (2.18), (2.21), (2.22), and the fact that

$$
\operatorname{tr} \Lambda_{1}=(\alpha+\beta) \frac{e^{t}}{2 \pi i} \int_{C} \frac{n d z^{\prime}}{z^{\prime}\left(z^{\prime}-e^{t}\right)}=-n(\alpha+\beta),
$$

we easily obtain

$$
\operatorname{tr}\left(\left(I-K_{n}\right)^{-1} K_{n}^{(1)}\right)=(\alpha+\beta) e^{t}\left(Y_{11}\left(e^{t}\right) Y_{z 22}^{\prime}\left(e^{t}\right)-Y_{21}\left(e^{t}\right) Y_{z 12}^{\prime}\left(e^{t}\right)\right),
$$

where $Y_{z}^{\prime}\left(e^{t}\right)$ stands for the derivative of $Y(z)$ w.r.t. $z$ evaluated at $z=e^{t}$.

Let us now compute the contribution of $K_{n}^{(2)}$. First, write $K_{n}^{(2)}$ in the form

$$
K_{n}^{(2)}=\Lambda_{2}-\frac{\alpha-\beta}{z^{\prime}-e^{-t}} \frac{\left(z / z^{\prime}\right)^{n}-1}{z-z^{\prime}} \frac{1-f\left(z^{\prime}\right)}{2 \pi i} e^{-t}, \quad \Lambda_{2}=\frac{\alpha-\beta}{z^{\prime}-e^{-t}} \frac{\left(z / z^{\prime}\right)^{n}-1}{z-z^{\prime}} \frac{1}{2 \pi i} e^{-t} .
$$

We then obtain as above for $K_{n}^{(1)}$ that

$$
K_{n}^{(2)}\left(z, z^{\prime}\right)=\left(\Lambda_{2}\left(I-K_{n}\right)\right)\left(z, z^{\prime}\right)+(\alpha-\beta) e^{-t} \frac{1-f\left(z^{\prime}\right)}{2 \pi i} \frac{\left(e^{-t} / z^{\prime}\right)^{n}-\left(z / z^{\prime}\right)^{n}}{\left(z-e^{-t}\right)\left(z^{\prime}-e^{-t}\right)} .
$$

Defining the new vectors

$$
\tilde{f}(z)=\frac{\hat{f}(z)}{z-e^{-t}}, \quad \widetilde{g}(z)=\frac{\hat{g}(z)}{z-e^{-t}}, \quad \widetilde{F}_{k}=(I-K)^{-1} \widetilde{f}_{k},
$$

we can write (2.24) in the form:

$$
K_{n}^{(2)}\left(z, z^{\prime}\right)=\left(\Lambda_{2}\left(I-K_{n}\right)\right)\left(z, z^{\prime}\right)+(\alpha-\beta) e^{-t}\left(e^{-n t} \widetilde{f}_{2}(z) \widetilde{g}_{1}\left(z^{\prime}\right)-\widetilde{f}_{1}(z) \widetilde{g}_{1}\left(z^{\prime}\right)\right)
$$

and obtain as above

$$
\widetilde{F}(z)=\frac{1}{z-e^{-t}}\left(\begin{array}{c}
m_{22}\left(e^{-t}\right) F_{1}(z)-m_{12}\left(e^{-t}\right) F_{2}(z) \\
-m_{21}\left(e^{-t}\right) F_{1}(z)+m_{11}\left(e^{-t}\right) F_{2}(z)
\end{array}\right) .
$$

For $|z|<1$, the matrix $m$ is related to $Y$ by the formula preceeding (3.21) in [24]:

$$
Y(z)=\left(\begin{array}{cc}
m_{11} z^{n}+m_{12} & m_{11} \\
-m_{21} z^{n}-m_{22} & -m_{21}
\end{array}\right)
$$

Using the definition $\widetilde{F}=\left(I-K_{n}\right)^{-1} \widetilde{f}$, equations (2.25), (2.26), (2.27), and the fact that $\operatorname{tr} \Lambda_{2}=0$, we finally obtain

$$
\operatorname{tr}\left(\left(I-K_{n}\right)^{-1} K_{n}^{(2)}\right)=(\alpha-\beta) e^{-t}\left(Y_{11}\left(e^{-t}\right) Y_{z 22}^{\prime}\left(e^{-t}\right)-Y_{21}\left(e^{-t}\right) Y_{z 12}^{\prime}\left(e^{-t}\right)\right) .
$$

Expressions (2.23) and (2.28) imply by (2.13) the statement of the Proposition. 


\section{Asymptotic analysis of the RH problem for orthogonal polynomials}

In this section, we apply the steepest descent method of Deift and Zhou [14] to the RH problem for $Y$. We follow the strategy used in [4] for orthogonal polynomials on the unit circle with a special weight function, and used in [12, 13] for orthogonal polynomials on the real line with respect to a more general weight function. The most important new feature here is the construction of a local parametrix near 1 which involves a Painlevé $\mathrm{V}$ RH problem. We will obtain asymptotics for $Y$ as $n \rightarrow \infty$, and therefore, asymptotics of the r.h.s. of (2.8) in terms of Painlevé V functions.

\subsection{Normalization of the RH problem}

Define

$$
T(z)= \begin{cases}Y(z) z^{-n \sigma_{3}}, & \text { as }|z|>1, \\ Y(z), & \text { as }|z|<1\end{cases}
$$

with $Y$ given by (2.7). Then $T$ satisfies a $\mathrm{RH}$ problem normalized at infinity.

\section{RH problem for $T$}

(a) $T: \mathbb{C} \backslash C \rightarrow \mathbb{C}^{2 \times 2}$ is analytic.

(b) $T_{+}(z)=T_{-}(z)\left(\begin{array}{cc}z^{n} & f(z) \\ 0 & z^{-n}\end{array}\right), \quad$ for $z \in C$.

(c) $T(z)=I+\mathcal{O}(1 / z) \quad$ as $z \rightarrow \infty$.

The diagonal elements of the jump matrix for $T$ oscillate rapidly on the unit circle if $n$ is large. The next transformation turns the oscillatory behavior into exponential decay on a deformed contour.

\subsection{Opening of the lens}

Note that one can factorize the jump matrix for $T$ as follows,

$$
\begin{aligned}
J_{T}(z) & :=\left(\begin{array}{cc}
z^{n} & f(z) \\
0 & z^{-n}
\end{array}\right) \\
& =\left(\begin{array}{cc}
1 & 0 \\
z^{-n} f(z)^{-1} & 1
\end{array}\right)\left(\begin{array}{cc}
0 & f(z) \\
-f(z)^{-1} & 0
\end{array}\right)\left(\begin{array}{cc}
1 & 0 \\
z^{n} f(z)^{-1} & 1
\end{array}\right) .
\end{aligned}
$$

To make use of this factorization, consider the three counterclockwise oriented closed curves as shown in Figure 2, Let us write

$$
\begin{aligned}
& J_{1}(z)=\left(\begin{array}{cc}
1 & 0 \\
z^{-n} f(z)^{-1} & 1
\end{array}\right), \\
& J_{N}(z)=\left(\begin{array}{cc}
0 & f(z) \\
-f(z)^{-1} & 0
\end{array}\right), \\
& J_{2}(z)=\left(\begin{array}{cc}
1 & 0 \\
z^{n} f(z)^{-1} & 1
\end{array}\right),
\end{aligned}
$$




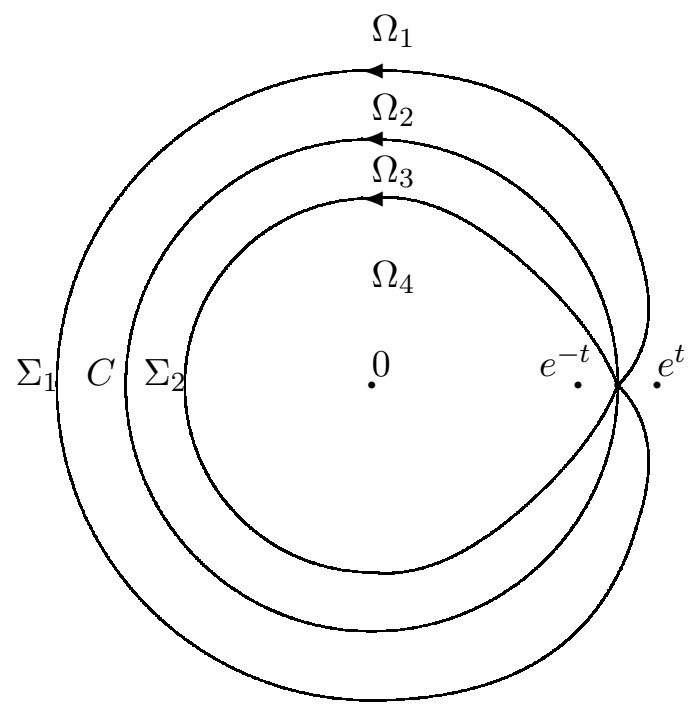

Figure 2: The contour $\Sigma=\Sigma_{1} \cup C \cup \Sigma_{2}$ and the regions $\Omega_{1}, \ldots, \Omega_{4}$.

and define

$$
S(z)= \begin{cases}T(z), & \text { in regions } \Omega_{1} \text { and } \Omega_{4}, \\ T(z) J_{1}, & \text { in region } \Omega_{2}, \\ T(z) J_{2}^{-1}, & \text { in region } \Omega_{3},\end{cases}
$$

with $\Omega_{1}, \Omega_{2}, \Omega_{3}$ as indicated in Figure 2, Note that $f$ is an analytic function in $\mathbb{C} \backslash$ $\left(\left[0, e^{-t}\right] \cup\left[e^{t},+\infty\right)\right)$, and we define $\Sigma_{1}, \Sigma_{2}$ so that the branch cuts are located in the regions $\Omega_{1}$ and $\Omega_{4}$. As we can have $t \rightarrow 0$, it is inevitable that $\Omega_{1}$ and $\Omega_{4}$ approach 1 . We choose $\Sigma_{1}$ and $\Sigma_{2}$ to go through 1 as in Figure 2, The function $S(z)$ satisfies the following $\mathrm{RH}$ problem.

\section{RH problem for $S$}

(a) $S: \mathbb{C} \backslash\left(\Sigma_{1} \cup C \cup \Sigma_{2}\right) \rightarrow \mathbb{C}^{2 \times 2}$ is analytic.

(b) $S_{+}(z)=S_{-}(z) J_{k}(z), \quad$ for $z \in \Sigma_{k}, k=1,2$, $S_{+}(z)=S_{-}(z) J_{N}(z), \quad$ for $z \in C$.

(c) $S(z)=I+\mathcal{O}(1 / z), \quad$ as $z \rightarrow \infty$.

Note that the jump matrices $J_{1}$ and $J_{2}$ tend to the identity matrix on their respective contours $\Sigma_{1}$ and $\Sigma_{2}$ as $n \rightarrow \infty$ except near 1 .

We need to construct a parametrix dealing with the jump condition on the unit circle and a local parametrix near 1.

\subsection{Global parametrix away from 1}

Ignoring the jumps on $\Sigma_{1}$ and $\Sigma_{2}$ and a neighborhood of 1 , we consider the following model problem.

\section{RH problem for $N$}

(a) $N: \mathbb{C} \backslash C \rightarrow \mathbb{C}^{2 \times 2}$ is analytic.

(b) $N_{+}(z)=N_{-}(z) J_{N}(z), \quad$ for $z \in C$. 
(c) $N(z)=I+\mathcal{O}(1 / z), \quad$ as $z \rightarrow \infty$.

This problem is easily solved explicitly:

$$
N(z)= \begin{cases}D(z)^{\sigma_{3}}\left(\begin{array}{cc}
0 & 1 \\
-1 & 0
\end{array}\right), & \text { for }|z|<1 \\
D(z)^{\sigma_{3}}, & \text { for }|z|>1\end{cases}
$$

where the (Szegö) function $D(z)$ is analytic and nonzero in $\mathbb{C} \backslash C$, tends to 1 as $z \rightarrow \infty$, and satisfies the jump condition $D_{+}(z)=D_{-}(z) f(z)$ for $z \in C$. It is easy to verify that

$$
D(z)= \begin{cases}\left(z-e^{t}\right)^{\alpha+\beta} e^{-i \pi(\alpha+\beta)} \exp \left(\sum_{k=0}^{\infty} V_{k} z^{k}\right), & \text { for }|z|<1 \\ \left(z-e^{-t}\right)^{-\alpha+\beta} z^{\alpha-\beta} \exp \left(-\sum_{k=-\infty}^{-1} V_{k} z^{k}\right), & \text { for }|z|>1\end{cases}
$$

\subsection{Local parametrix near 1}

For $0<t<t_{0}$ with $t_{0}$ fixed but sufficiently small, we will now construct a parametrix $P$ satisfying the same jump conditions as $S$ in a neighborhood $U$ of 1 of a sufficiently small fixed radius and a matching condition with $N$ on the boundary $\partial U$.

Assume that $\Psi(\zeta)$ solves the RH problem of Section 1.3, and define

$$
\Phi(\lambda ; x)=e^{\frac{x}{4} \sigma_{3}} x^{-\beta \sigma_{3}} \Psi\left(\frac{\lambda}{x}+\frac{1}{2} ; x\right) G(\lambda ; x)^{\frac{1}{2} \sigma_{3}} e^{ \pm \frac{\pi i}{2}(\alpha-\beta) \sigma_{3}}, \quad \text { for } \pm \operatorname{Im} \lambda>0
$$

respectively, with

$$
G(\lambda ; x)=\left(\lambda+\frac{x}{2}\right)^{-(\alpha-\beta)}\left(\lambda-\frac{x}{2}\right)^{\alpha+\beta} e^{\lambda} e^{-\pi i(\alpha-\beta)}, \quad x>0,
$$

where $G$ is analytic in $\mathbb{C} \backslash\left(\left(-\infty,-\frac{x}{2}\right] \cup\left[\frac{x}{2},+\infty\right)\right)$. We choose $-\pi<\arg \left(\lambda+\frac{x}{2}\right)<\pi$ and $0<\arg \left(\lambda-\frac{x}{2}\right)<2 \pi$. It is straightforward to check that $\Phi=\Phi(\lambda ; x)$ solves the following RH problem for $x>0$.

\section{RH problem for $\Phi$}

(a) $\Phi: \mathbb{C} \backslash \cup_{j=1}^{4} e^{\frac{\pi i(2 j-1)}{4}} \mathbb{R}^{+} \rightarrow \mathbb{C}^{2 \times 2}$ is analytic, with the rays $e^{\frac{\pi i(2 j-1)}{4}} \mathbb{R}^{+}$oriented as shown in Figure 3 .

(b) $\Phi$ has continuous boundary values on $\cup_{j=1}^{4} e^{\frac{\pi i(2 j-1)}{4}} \mathbb{R}^{+} \backslash\{0\}$, and they are related by the jump conditions:

$$
\begin{array}{ll}
\Phi_{+}(\lambda)=\Phi_{-}(\lambda)\left(\begin{array}{cc}
1 & G(\lambda ; x)^{-1} \\
0 & 1
\end{array}\right), & \text { as } \lambda \in e^{\frac{\pi i}{4}} \mathbb{R}^{+} \cup e^{\frac{7 \pi i}{4}} \mathbb{R}^{+}, \\
\Phi_{+}(\lambda)=\Phi_{-}(\lambda)\left(\begin{array}{cc}
1 & 0 \\
-G(\lambda ; x) & 1
\end{array}\right), & \text { as } \lambda \in e^{\frac{3 \pi i}{4}} \mathbb{R}^{+} \cup e^{\frac{5 \pi i}{4}} \mathbb{R}^{+} .
\end{array}
$$

(c) $\Phi$ has the following behavior as $\lambda \rightarrow \infty$ :

$$
\Phi(\lambda)=I+\mathcal{O}\left(\lambda^{-1}\right) .
$$

(d) $\Phi$ is bounded near 0 . 


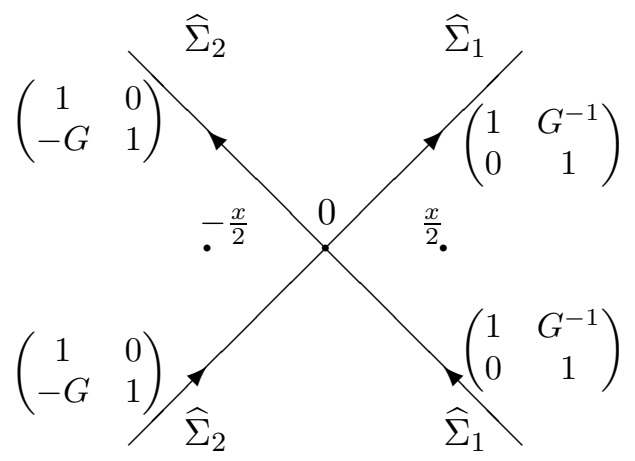

Figure 3: The jump contour and jump matrices for $\Phi$.

We will prove the following results.

Proposition $3.1 \quad$ (i) If $\operatorname{Re} \alpha>-\frac{1}{2}$, the RH problem for $\Phi$ is uniquely solvable for all but possibly a finite number of positive $x$-values $\left\{x_{1}, \ldots, x_{k}\right\}$, where $x_{j}=x_{j}(\alpha, \beta)$ and $k=k(\alpha, \beta)$.

(ii) If $\alpha>-\frac{1}{2}(\operatorname{Im} \alpha=0)$ and $\operatorname{Re} \beta=0$, the $R H$ problem for $\Phi$ is (uniquely) solvable for all $x>0$.

(iii) If $\operatorname{Re} \alpha>-\frac{1}{2}$, the asymptotic condition (3.13) for $\Phi$ is valid uniformly for $x \in$ $(0,+\infty)$ provided that $x$ remains bounded away from the set $\left\{x_{1}, \ldots, x_{k}\right\}$.

Statements (i) and (ii) follow immediately from Theorem 1.8 (which will be proven in Section (4). The third statement will follow from our asymptotic analysis of the RH problem for $\Phi$ in Section 4 .

We will now transform the jump matrices for $\Phi$ into the jump matrices for $S$ near 1 . Note first that the off-diagonal entries of the jump matrices for $\Phi$ have branch points at $\pm \frac{x}{2}$, and the ones for $S$ at $e^{ \pm t}$. Let us therefore define a conformal mapping $\lambda(z)$ in a neighborhood of 1 which maps $e^{-t}$ to $-\frac{x}{2}, e^{t}$ to $\frac{x}{2}$, and 1 to 0 :

$$
\lambda(z)=\frac{x}{2 t} \ln (z), \quad z \in U .
$$

Here we take the branch of the logarithm such that $\ln z>0$ for $z>1$, and the branch cut is along the negative real axis. We will furthermore need that $e^{\lambda(z)}=z^{n}$, and therefore set

$$
x=2 n t .
$$

Let us choose the contours $\Sigma_{1}$ and $\Sigma_{2}$ near 1 in such a way that $\lambda$ maps $\Sigma_{1} \cup \Sigma_{2}$ onto the jump contour $\cup_{j=1}^{4} e^{\frac{\pi i(2 j-1)}{4}} \mathbb{R}^{+}$for $\Phi$. We look for the parametrix $P$ in the form

$$
P(z)=E(z) \Phi(\lambda(z) ; 2 n t) W(z)
$$

where $E$ is an analytic function in $U$, and $W$ is given by

$$
W(z)= \begin{cases}-G(\lambda(z))^{-\frac{1}{2} \sigma_{3}} z^{\frac{n}{2} \sigma_{3}} f(z)^{-\frac{1}{2} \sigma_{3}} \sigma_{3}, & \text { for }|z|<1, \\ G(\lambda(z))^{-\frac{1}{2} \sigma_{3}} z^{\frac{n}{2} \sigma_{3}} f(z)^{\frac{1}{2} \sigma_{3}} \sigma_{1}, & \text { for }|z|>1,\end{cases}
$$


with $\sigma_{1}=\left(\begin{array}{ll}0 & 1 \\ 1 & 0\end{array}\right)$. Note that the branch points of $G$ cancel the ones for $f$ in $U$, and $W$ is analytic in $U \backslash C$.

If $E$ is analytic in $U$, it is easy to check using (3.11)-(3.12) that $P(z)$ satisfies the same jump conditions as the matrix $S$ with the jump matrices given in (3.3)-(3.5). Since we evaluate $\Phi(\lambda ; x)$ at $x=2 n t$, we need to impose the condition that $2 n t$ does not belong to the set $\left\{x_{1}, \ldots, x_{k}\right\}$ of values at which the RH problem for $\Phi$ is not solvable.

To fix $E(z)$, let us consider the behavior of $P$ on $\partial U$. From (3.14) one observes that there exists $c>0$ such that for any $0<t<t_{0}$

$$
|\lambda(z)|>c n, \quad z \in \partial U
$$

As $n \rightarrow \infty$ and if $2 n t$ stays bounded away from the set $\left\{x_{1}, \ldots, x_{k}\right\}$, we can thus (by Proposition 3.1) use the asymptotic behavior (3.13) for $\Phi$ to conclude that

$$
P(z)=E(z)\left(I+\mathcal{O}\left(n^{-1}\right)\right) W(z), \quad \text { as } n \rightarrow \infty,
$$

uniformly for $0<t<t_{0}$ and $z \in \partial U$. If $t_{0}$ is sufficiently small, we can assume that $e^{ \pm t}$ lie inside $U$ and at a distance bounded from below away from $\partial U$. Then we obtain from (3.17) and (3.10) that (here and in (3.22) below, $\mathcal{O}(1)$ is a scalar matrix element)

$$
W(z)=n^{-\beta \sigma_{3}}\left\{\begin{array}{cc}
\left(\begin{array}{cc}
\mathcal{O}(1) & 0 \\
0 & \mathcal{O}(1)
\end{array}\right), & |z|<1 \\
0 & \mathcal{O}(1) \\
\mathcal{O}(1) & 0
\end{array}\right), \quad|z|>1
$$

as $n \rightarrow \infty$ uniformly for $0<t<t_{0}$ and uniformly for $z \in \partial U \backslash C$.

Now set

$$
E(z)=N(z) W(z)^{-1} .
$$

One verifies directly, using the jumps for $N$ and $W$ across $C$, that $E$ is analytic in a full neighborhood $\bar{U}$ of 1 . Furthermore, by (3.20), (3.7),

$$
E(z)=\left(\begin{array}{cc}
0 & \mathcal{O}(1) \\
\mathcal{O}(1) & 0
\end{array}\right) n^{\beta \sigma_{3}}
$$

as $n \rightarrow \infty$ uniformly for $0<t<t_{0}$ and $z \in \partial U$.

Using this result and (3.19), we obtain the following matching condition on $z \in \partial U$ :

$$
P(z) N(z)^{-1}=E(z)\left(I+\mathcal{O}\left(n^{-1}\right)\right) E(z)^{-1}=I+n^{-\beta \sigma_{3}} \mathcal{O}\left(n^{-1}\right) n^{\beta \sigma_{3}} .
$$

as $n \rightarrow \infty$ uniformly for $0<t<t_{0}$ and $z \in \partial U$. Note once again that the matching holds true if $2 n t$ remains bounded away from the set $\left\{x_{1}, \ldots, x_{k}\right\}$.

Since $P$ has the same jumps as $S$ inside $U$ and $S(z) P(z)^{-1}=\mathcal{O}(\ln (z-1))$ as $z \rightarrow 1$ for $t>0$, it follows that the singularity is removable and $S(z) P(z)^{-1}$ is analytic in $U$. For later use, we note that

$$
E\left(e^{t}\right)=n^{-\beta \sigma_{3}} g^{\sigma_{3}} \sigma_{1}, \quad E\left(e^{-t}\right)=n^{-\beta \sigma_{3}} h^{\sigma_{3}} \sigma_{1},
$$




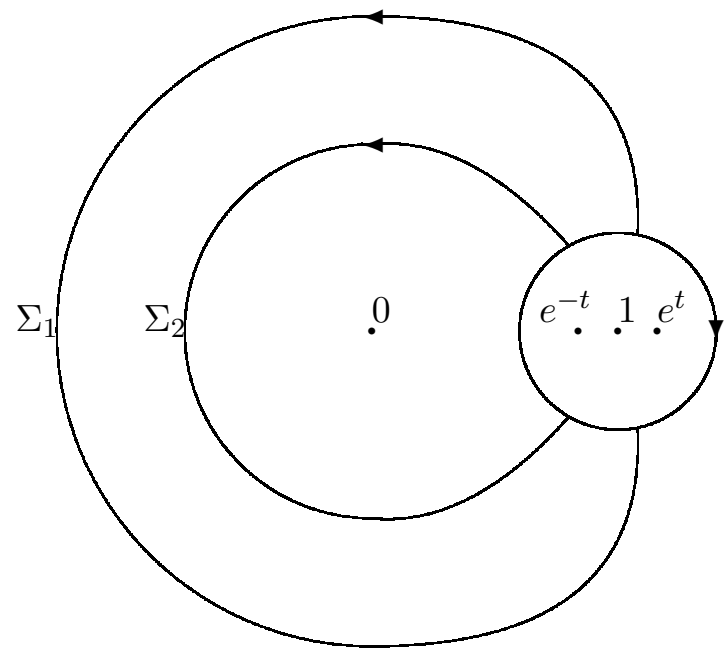

Figure 4: The contour $\Sigma_{R}$.

with $g$ and $h$ given by

$$
\begin{aligned}
& g=\left(\frac{\sinh t}{t}\right)^{-\frac{\alpha-\beta}{2}} e^{-\pi i \beta} e^{t \alpha} \exp \left(-\frac{1}{2} \sum_{k=-\infty}^{-1} V_{k} e^{t k}+\frac{1}{2} \sum_{k=0}^{\infty} V_{k} e^{t k}\right), \\
& h=\left(\frac{\sinh t}{t}\right)^{\frac{\alpha+\beta}{2}} e^{-\pi i \beta} \exp \left(-\frac{1}{2} \sum_{k=-\infty}^{-1} V_{k} e^{-t k}+\frac{1}{2} \sum_{k=0}^{\infty} V_{k} e^{-t k}\right) .
\end{aligned}
$$

\subsection{Final RH problem}

Define

$$
R(z)= \begin{cases}n^{\beta \sigma_{3}} S(z) N(z)^{-1} n^{-\beta \sigma_{3}}, & \text { for } z \in \mathbb{C} \backslash U, \\ n^{\beta \sigma_{3}} S(z) P(z)^{-1} n^{-\beta \sigma_{3}}, & \text { for } z \in U .\end{cases}
$$

Using the RH properties of $S, N$, and $P$, we obtain the following.

\section{RH problem for $R$}

(a) $R$ is analytic in $\mathbb{C} \backslash \Sigma_{R}$, where $\Sigma_{R}$ is the union of $\partial U$ and the parts of $\Sigma_{1}, \Sigma_{2}$ lying outside $U$ (see Figure 4).

(b) $R_{+}(z)=R_{-}(z) J_{R}(z)$ for $z \in \Sigma_{R}$, where

$$
\begin{array}{lr}
J_{R}(z)=n^{\beta \sigma_{3}} P(z) N(z)^{-1} n^{-\beta \sigma_{3}}, & \text { for } z \in \partial U, \\
J_{R}(z)=n^{\beta \sigma_{3}} N(z) J_{k}(z) N(z)^{-1} n^{-\beta \sigma_{3}}, & \text { for } z \in \Sigma_{k} \text { outside } U,
\end{array}
$$

and $J_{k}(z), k=1,2$ are the jump matrices (3.3), (3.5) of $S$.

(c) As $z \rightarrow \infty, R(z)=I+\mathcal{O}\left(z^{-1}\right)$.

Using (3.3), (3.5), and (3.23), we observe a crucial fact:

$$
\begin{array}{lr}
J_{R}(z)=I+\mathcal{O}\left(n^{-1}\right), & \text { for } z \in \partial U, \\
J_{R}(z)=I+\mathcal{O}\left(e^{-c n}\right), & \text { for } z \in \Sigma_{R} \backslash \partial U, c>0
\end{array}
$$


as $n \rightarrow \infty$ uniformly in $z$ and uniformly for $0<t<t_{0}$ as long as $2 n t$ remains bounded away from the set $\left\{x_{1}, \ldots, x_{k}\right\}$. Thus the jump matrix $J_{R}$ tends to the identity matrix as $n \rightarrow \infty$. The RH problem for $R$ is therefore a so-called small-norm RH problem, and by a standard analysis (see, e.g., [13]) we obtain the following statement.

Proposition 3.2 Let $0<t<t_{0}$. Then

(i) The $R H$ problem for $R$ is solvable for $n$ sufficiently large as long as 2 nt remains bounded away from the set $\left\{x_{1}, \ldots, x_{k}\right\}$.

(ii) If $n \rightarrow \infty$,

$$
R(z)=I+\mathcal{O}\left(n^{-1}\right), \quad \text { uniformly for } z \in \mathbb{C} \backslash \Sigma_{R}
$$

and for $0<t<t_{0}$ such that 2 t remains bounded away from the set $\left\{x_{1}, \ldots, x_{k}\right\}$.

\subsection{Asymptotics for $\frac{d}{d t} \ln D_{n}$}

Reversing the transformations $S \mapsto R, T \mapsto S$, and $Y \mapsto T$ by (3.1), (3.6), and (3.27), we obtain the asymptotics for $Y(z)$ under the conditions of Proposition 3.2. In particular, we obtain

$$
\begin{array}{lrl}
Y(z) & =n^{-\beta \sigma_{3}}\left(I+\mathcal{O}\left(n^{-1}\right)\right) n^{\beta \sigma_{3}} P(z) z^{n \sigma_{3}}, & \text { for } z \text { near } e^{t}, \\
Y(z)=n^{-\beta \sigma_{3}}\left(I+\mathcal{O}\left(n^{-1}\right)\right) n^{\beta \sigma_{3}} P(z), & \text { for } z \text { near } e^{-t} .
\end{array}
$$

as $n \rightarrow \infty$, uniformly for $0<t<t_{0}$ if $2 n t$ remains bounded away from $\left\{x_{1}, \ldots, x_{k}\right\}$. Using the definitions (3.7), (3.16), and (3.24), we find for $P$ in the above formulas:

$$
\begin{aligned}
& P(z)=E(z) \Phi(z) W(z)=D(z)^{\sigma_{3}} W(z)^{-1} \Phi(z) W(z), \quad \text { for } z \text { near } e^{t}, \\
& P(z)=E(z) \Phi(z) W(z)=D(z)^{\sigma_{3}}\left(\begin{array}{cr}
0 & 1 \\
-1 & 0
\end{array}\right) W(z)^{-1} \Phi(z) W(z), \quad \text { for } z \text { near } e^{-t} .
\end{aligned}
$$

We will now substitute the asymptotics we obtained for $Y$ into the differential identity (2.8) for $\ln D_{n}(t)$. First, consider the case of $z$ close to $e^{t}$. By (3.33), we obtain $Y^{-1} Y_{z}^{\prime}=\frac{n \sigma_{3}}{z}+z^{-n \sigma_{3}} P^{-1} P_{z}^{\prime} z^{n \sigma_{3}}+z^{-n \sigma_{3}} P^{-1}(z) n^{-\beta \sigma_{3}}\left(I+\mathcal{O}\left(n^{-1}\right)\right)^{-1} \mathcal{O}\left(n^{-1}\right)_{z}^{\prime} n^{\beta \sigma_{3}} P(z) z^{n \sigma_{3}}$.

Using (3.35) and (3.17), we further obtain

$$
P^{-1} P_{z}^{\prime}=-\sigma_{3} \frac{A_{z}^{\prime}}{A}+W^{-1} \Phi^{-1} \Phi_{z}^{\prime} W-W^{-1} \Phi^{-1} \sigma_{3} \Phi W\left(\frac{A_{z}^{\prime}}{A}+\frac{D_{z}^{\prime}}{D}\right)
$$

where we defined $A(z)$ by the formula

$$
W(z)=A(z)^{\sigma_{3}} \sigma_{1} .
$$

Expressions (3.17) and (3.8) give

$$
\begin{aligned}
& \frac{A_{z}^{\prime}}{A}\left(e^{t}\right)=\frac{\alpha+\beta}{4} e^{-t}+\frac{\alpha-\beta}{4} e^{-t}\left(\frac{1}{t}+\frac{e^{-t}}{\sinh t}\right)+\frac{1}{2} V_{z}^{\prime}\left(e^{t}\right) ; \\
& \frac{D_{z}^{\prime}}{D}\left(e^{t}\right)=-\frac{\alpha-\beta}{2 \sinh t} e^{-2 t}-\sum_{k=-\infty}^{-1} k V_{k} e^{(k-1) t}
\end{aligned}
$$


Therefore, we finally have for the 22 matrix element of $P^{-1} P_{z}^{\prime}$ at the point $e^{t}$ :

$$
\begin{aligned}
& e^{t}\left(P^{-1} P_{z}^{\prime}\right)_{22}\left(e^{t}\right)=\frac{\alpha+\beta}{4}+\frac{\alpha-\beta}{4}\left(\frac{1}{t}+\frac{e^{-t}}{\sinh t}\right)+\frac{1}{2} e^{t} V_{z}^{\prime}\left(e^{t}\right)+e^{t}\left(\Phi^{-1} \Phi_{z}^{\prime}\right)_{11}\left(e^{t}\right)- \\
& \left(\frac{\alpha+\beta}{4}+\frac{\alpha-\beta}{4}\left(\frac{1}{t}-\frac{e^{-t}}{\sinh t}\right)+\frac{1}{2} e^{t} V_{z}^{\prime}\left(e^{t}\right)-\sum_{k=-\infty}^{-1} k V_{k} e^{k t}\right)\left(\Phi^{-1} \sigma_{3} \Phi\right)_{11}\left(e^{t}\right) .
\end{aligned}
$$

Now using the definition of $W(z)$ it is easy to conclude that

$$
n^{\beta \sigma_{3}} P\left(e^{t}\right)=\hat{\Phi}(t) n^{\beta \sigma_{3}},
$$

where $\hat{\Phi}(t)$ is bounded in $n$ as long as $\Phi\left(\frac{x}{2}\right)$ is bounded. Thus, we obtained the asymptotic expression

$$
e^{t}\left(Y^{-1} Y_{z}^{\prime}\right)_{22}\left(e^{t}\right)=-n+e^{t}\left(P^{-1} P_{z}^{\prime}\right)_{22}\left(e^{t}\right)+\left(\hat{\Phi}^{-1}(t) \mathcal{O}(1 / n) \hat{\Phi}(t)\right)_{22}
$$

uniformly for $0<t<t_{0}$ as long as $2 n t$ remains bounded away from the set $\left\{x_{1}, \ldots, x_{k}\right\}$, with the second term on the r.h.s. given by (3.41).

Similar calculations at $e^{-t}$ give

$$
e^{-t}\left(Y^{-1} Y_{z}^{\prime}\right)_{22}\left(e^{-t}\right)=e^{-t}\left(P^{-1} P_{z}^{\prime}\right)_{22}\left(e^{-t}\right)+\left(\hat{\Phi}^{-1}(t) \mathcal{O}(1 / n) \hat{\Phi}(t)\right)_{22},
$$

with

$$
\begin{aligned}
& e^{-t}\left(P^{-1} P_{z}^{\prime}\right)_{22}\left(e^{-t}\right)=-\frac{\alpha-\beta}{4}-\frac{\alpha+\beta}{4}\left(\frac{1}{t}+\frac{e^{-t}}{\sinh t}\right)+\frac{1}{2} e^{-t} V_{z}^{\prime}\left(e^{-t}\right)+e^{-t}\left(\Phi^{-1} \Phi_{z}^{\prime}\right)_{22}\left(e^{-t}\right)- \\
& \left(\frac{\alpha-\beta}{4}+\frac{\alpha+\beta}{4}\left(\frac{1}{t}-\frac{e^{-t}}{\sinh t}\right)-\frac{1}{2} e^{-t} V_{z}^{\prime}\left(e^{-t}\right)+\sum_{k=1}^{\infty} k V_{k} e^{-k t}\right)\left(\Phi^{-1} \sigma_{3} \Phi\right)_{22}\left(e^{-t}\right) .
\end{aligned}
$$

Collecting (3.42) and (3.43) together, substituting into (2.8), and noting that

$$
\Phi_{z}^{\prime}=\frac{n}{z} \Phi_{\lambda}^{\prime}
$$

gives

\section{Proposition 3.3 Let}

$$
w(x)=-\frac{\alpha+\beta}{2}\left(\Phi^{-1} \Phi_{\lambda}^{\prime}\right)_{11}(x / 2)+\frac{\alpha-\beta}{2}\left(\Phi^{-1} \Phi_{\lambda}^{\prime}\right)_{22}(-x / 2) .
$$

Then

$$
\begin{aligned}
& \frac{d}{d t} \ln D_{n}=(\alpha+\beta) n-\frac{\alpha^{2}+\beta^{2}}{2}-\frac{\alpha^{2}-\beta^{2}}{2}\left(\frac{1}{t}+\frac{e^{-t}}{\sinh t}\right) \\
& -\frac{\alpha+\beta}{2} e^{t} V_{z}^{\prime}\left(e^{t}\right)+\frac{\alpha-\beta}{2} e^{-t} V_{z}^{\prime}\left(e^{-t}\right)+2 n w(x) \\
& +\frac{\alpha+\beta}{2}\left\{\frac{\alpha+\beta}{2}+\frac{\alpha-\beta}{2}\left(\frac{1}{t}-\frac{e^{-t}}{\sinh t}\right)+\sum_{k=1}^{\infty} k\left(V_{k} e^{k t}+V_{-k} e^{-k t}\right)\right\}\left(\Phi^{-1} \sigma_{3} \Phi\right)_{11}\left(e^{t}\right) \\
& -\frac{\alpha-\beta}{2}\left\{\frac{\alpha-\beta}{2}+\frac{\alpha+\beta}{2}\left(\frac{1}{t}-\frac{e^{-t}}{\sinh t}\right)+\sum_{k=1}^{\infty} k\left(V_{k} e^{-k t}+V_{-k} e^{k t}\right)\right\}\left(\Phi^{-1} \sigma_{3} \Phi\right)_{22}\left(e^{-t}\right) \\
& +O(1 / n) \widetilde{\Phi}(x),
\end{aligned}
$$


where the error term is uniform for $0<t<t_{0}$ as long as 2 nt remains bounded away from the set $\left\{x_{1}, \ldots, x_{k}\right\}$, and $\widetilde{\Phi}(x)$ depends on $\Phi(x / 2), \Phi(-x / 2), \alpha, \beta$ only and is bounded when these parameters are in a compact set.

In the next section, we will analyze the Painlevé functions $\Phi(\lambda)$. Namely, we will obtain their behavior at $x=0$ and $\infty$ which will be used in the last section to prove Theorems 1.1 and 1.4 .

\section{Model RH problem near $z=1$ and the fifth Painlevé equation}

Recall the RH problem for $\Psi$ stated in the introduction on the contour $\Gamma$ given in Figure 1 for $x>0$ and $\operatorname{Re} \alpha>-\frac{1}{2}$. In this section, we will analyze the $\Psi$-RH problem asymptotically for $x$ near zero and infinity and prove Theorem 1.8 and Proposition 3.1 . Moreover, we will give an explicit formula for $w$ (defined by (3.45) ) in terms of the Painlevé V function $v$.

For simplicity, we will omit the dependence of $\Psi$ on $\alpha, \beta$, and $x$ in our notation when convenient. The behavior of $\Psi$ near 0 and 1 can also be characterized in a different way from conditions (1.39), (1.40). The following statement holds.

Proposition 4.1 Let $\Psi=\Psi(\zeta ; x, \alpha, \beta)$ satisfy the conditions (a), (b), (d0), and (d1) of the $R H$ problem for $\Psi$. Set

$$
\Psi_{0}(\zeta):=\Psi(\zeta)(\zeta-1)^{\frac{\alpha+\beta}{2} \sigma_{3}} \zeta^{-\frac{\alpha-\beta}{2} \sigma_{3}} .
$$

Then the function $\Psi_{0}$ is analytic near 0 and near 1 . The branch cuts for $\zeta^{-\frac{\alpha-\beta}{2} \sigma_{3}}$ and $(\zeta-1)^{\frac{\alpha+\beta}{2} \sigma_{3}}$ are chosen here along $[0,+\infty)$ and $[1,+\infty)$, respectively.

Proof. The fact that $\Psi_{0}$ is analytic near 0 and 1 can be verified using the jump conditions for $\Psi$ for $(\zeta-1)^{\frac{\alpha+\beta}{2} \sigma_{3}}$ and $\zeta^{-\frac{\alpha-\beta}{2} \sigma_{3}}$. The isolated singularities at 0 and 1 are removable because of the conditions $(\mathrm{d} 0)$ and $(\mathrm{d} 1)$.

Recall the function $\Phi$ defined in terms of $\Psi$ in (3.9)-(3.10). It satisfies the RH problem given in Section 3.4 .

We will now perform an asymptotic analysis of the RH problem for $\Phi$ as $x \rightarrow+\infty$ and as $x \searrow 0$.

\subsection{Asymptotics for $\Phi$ as $x \rightarrow+\infty$}

Consider Figure 5 and define

$$
\widetilde{\Phi}(\zeta ; x)=\Phi(x \zeta ; x)
$$

for $\zeta$ outside of the two triangular regions $A$ and $B$. In these regions set

$$
\begin{array}{ll}
\widetilde{\Phi}(\zeta ; x)=\Phi(x \zeta ; x)\left(\begin{array}{cc}
1 & G(x \zeta ; x)^{-1} \\
0 & 1
\end{array}\right), & \text { in region } \mathrm{B}, \\
\widetilde{\Phi}(\zeta ; x)=\Phi(x \zeta ; x)\left(\begin{array}{cc}
1 & 0 \\
G(x \zeta ; x) & 1
\end{array}\right), & \text { in region A. }
\end{array}
$$

Now $\widetilde{\Phi}$ is defined in such a way that it has its jumps only on the solid lines in Figure 5. We have 


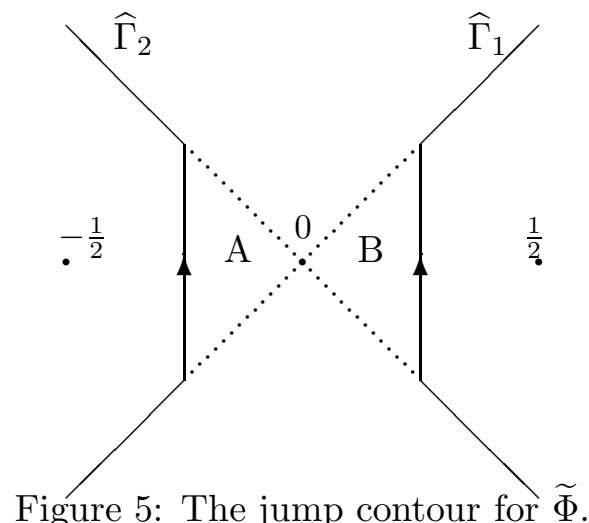

\section{RH problem for $\widetilde{\Phi}$}

(a) $\widetilde{\Phi}: \mathbb{C} \backslash\left(\widehat{\Gamma}_{1} \cup \widehat{\Gamma}_{2}\right) \rightarrow \mathbb{C}^{2 \times 2}$ is analytic.

(b) $\widetilde{\Phi}$ has continuous boundary values on $\widehat{\Gamma}_{1} \cup \widehat{\Gamma}_{2}$ related by the conditions:

$$
\begin{array}{ll}
\widetilde{\Phi}_{+}(\zeta)=\widetilde{\Phi}_{-}(\zeta)\left(\begin{array}{cc}
1 & G(x \zeta ; x)^{-1} \\
0 & 1
\end{array}\right), & \text { for } \zeta \in \widehat{\Gamma}_{1}, \\
\widetilde{\Phi}_{+}(\zeta)=\widetilde{\Phi}_{-}(\zeta)\left(\begin{array}{cc}
1 & 0 \\
-G(x \zeta ; x) & 1
\end{array}\right), & \text { as } \zeta \in \widehat{\Gamma}_{2} .
\end{array}
$$

(c) $\widetilde{\Phi}$ has the following behavior as $\zeta \rightarrow \infty$,

$$
\widetilde{\Phi}(\zeta)=I+\mathcal{O}\left(\zeta^{-1}\right) .
$$

The jump matrices for $\widetilde{\Phi}$ are exponentially close to $I$ as $x \rightarrow+\infty$ because of the exponential factor in the definition of $G$ (see (3.10) ). Indeed, let us denote $G_{\widetilde{\Phi}}(\zeta)$ the jump matrix for the function $\widetilde{\Phi}(z)$, i.e.

$$
G_{\widetilde{\Phi}}(\zeta)=\left(\begin{array}{cc}
1 & G(x \zeta ; x)^{-1} \\
0 & 1
\end{array}\right), \quad \zeta \in \widehat{\Gamma}_{1},
$$

and

$$
G_{\widetilde{\Phi}}(\zeta)=\left(\begin{array}{cc}
1 & 0 \\
-G(x \zeta ; x) & 1
\end{array}\right), \quad \zeta \in \widehat{\Gamma}_{2}
$$

Then, the following estimates hold:

$$
\left\|I-G_{\widetilde{\Phi}}\right\|_{L_{2}(\widehat{\Gamma})},\left\|I-G_{\widetilde{\Phi}}\right\|_{L_{\infty}(\widehat{\Gamma})}<C e^{-\frac{x}{2}(1-\epsilon)}, \quad 0<\epsilon<1, \quad C>0,
$$

where

$$
\widehat{\Gamma}=\widehat{\Gamma}_{1} \cup \widehat{\Gamma}_{2}
$$

and we assume that the vertical parts of the contours $\widehat{\Gamma}_{1}$ and $\widehat{\Gamma}_{2}$ are given by the equations $\operatorname{Re} \zeta=1 / 2-\epsilon / 2$ and $\operatorname{Re} \zeta=-1 / 2+\epsilon / 2$, respectively. Estimates (4.6) imply that the RH problem for $\widetilde{\Phi}$ is a small-norm RH problem for large $x$ and is therefore solvable in a standard way (see e.g., [13]) for $x$ sufficiently large. Moreover, the solution $\widetilde{\Phi}$ admits the integral representation,

$$
\widetilde{\Phi}(\zeta)=I+\frac{1}{2 \pi i} \int_{\widehat{\Gamma}} \rho\left(\zeta^{\prime}\right)\left(G_{\widetilde{\Phi}}\left(\zeta^{\prime}\right)-I\right) \frac{d \zeta^{\prime}}{\zeta^{\prime}-\zeta}
$$


with the function $\rho(\zeta)$ which is $L_{2}$ - close to the identity, namely,

$$
\|I-\rho\|_{L_{2}(\widehat{\Gamma})}<C e^{-\frac{x}{2}(1-\epsilon)} .
$$

The large $x$ solvability of the $\widetilde{\Phi}$-problem means that the RH problems for $\Phi$ and $\Psi$ are solvable for $x$ sufficiently large as well. In addition, we have that

$$
\widetilde{\Phi}(\zeta ; x)=I+\mathcal{O}\left(\frac{1}{\zeta} e^{-\frac{x}{2}(1-\epsilon)}\right), \quad \text { as } x \rightarrow+\infty .
$$

This estimate holds uniformly for $\zeta$ off the jump contour and this implies, in particular, that the following asymptotics hold as $x \rightarrow+\infty$ :

$$
\begin{aligned}
& \widetilde{\Phi}\left( \pm \frac{1}{2} ; x\right)=\Phi\left( \pm \frac{x}{2} ; x\right)=I+\mathcal{O}\left(e^{-\frac{x}{2}(1-\epsilon)}\right), \\
& \Phi_{\lambda}^{\prime}\left( \pm \frac{x}{2} ; x\right)=\mathcal{O}\left(e^{-\frac{x}{2}(1-\epsilon)}\right) .
\end{aligned}
$$

Furthermore, (4.9) implies that

$$
\Phi(\lambda ; x)=I+\mathcal{O}\left(\lambda^{-1}\right), \quad \text { as } \lambda \rightarrow \infty, \text { uniformly for } x>C, C>0 .
$$

The integral representation (4.7) in conjunction with the estimate (4.8) allows to evaluate the asymptotics for the Painlevé function $v(x)$ defined in (1.41). Let $\widetilde{C}_{1}$ be the first coefficient in the large $\zeta$ expansion of the function $\widetilde{\Phi}$,

$$
\widetilde{\Phi}(\zeta)=I+\frac{\widetilde{C}_{1}}{\zeta}+\mathcal{O}\left(\zeta^{-2}\right), \quad \zeta \rightarrow \infty .
$$

(Note that this expansion is uniform for $x>C, C>0$.) Recalling the relation between the functions $\widetilde{\Phi}, \Phi$, and $\Psi$, we obtain the following expression of the first coefficient $C_{1}$ of the series (1.38) in terms of the coefficient $\widetilde{C}_{1}$,

$$
C_{1}=\frac{\alpha+\beta}{2} \sigma_{3}+e^{-\frac{x}{4} \sigma_{3}} x^{\beta \sigma_{3}} \widetilde{C}_{1} x^{-\beta \sigma_{3}} e^{\frac{x}{4} \sigma_{3}} .
$$

Together with (1.41), this means that

$$
v(x)=-\widetilde{C}_{1,11}-x \widetilde{C}_{1,12} \widetilde{C}_{1,21} .
$$

On the other hand, from (4.7) we obtain

$$
\widetilde{C}_{1}=-\frac{1}{2 \pi i} \int_{\widehat{\Gamma}} \rho(\zeta)\left(G_{\widetilde{\Phi}}(\zeta)-I\right) d \zeta
$$

which leads to the estimate:

$$
\begin{aligned}
\widetilde{C}_{1} & =-\frac{1}{2 \pi i} \int_{\widehat{\Gamma}}\left(G_{\widetilde{\Phi}}(\zeta)-I\right) d \zeta-\frac{1}{2 \pi i} \int_{\widehat{\Gamma}}(\rho(\zeta)-I)\left(G_{\widetilde{\Phi}}(\zeta)-I\right) d \zeta \\
& =-\frac{1}{2 \pi i} \int_{\widehat{\Gamma}}\left(G_{\widetilde{\Phi}}(\zeta)-I\right) d \zeta+\mathcal{O}\left(e^{-x(1-\epsilon)}\right), \quad 0<\epsilon<\frac{1}{2} .
\end{aligned}
$$

The estimate (4.17) implies the following asymptotic representations for the entries of the matrix $\widetilde{C}_{1}$ as $x \rightarrow+\infty$ :

$$
\begin{aligned}
\widetilde{C}_{1,12} & =-\frac{1}{2 \pi i} \int_{\widehat{\Gamma}_{1}} G(x \zeta ; x)^{-1} d \zeta+\mathcal{O}\left(e^{-x(1-\epsilon)}\right) \\
& =-x^{-2 \beta} e^{-\frac{x}{2}} \frac{e^{-2 \pi i \beta}}{\Gamma(\alpha+\beta)} \psi(1-\alpha-\beta, 2-2 \beta ; x)+\mathcal{O}\left(e^{-x(1-\epsilon)}\right) \\
& =-x^{-1+\alpha-\beta} e^{-\frac{x}{2}} \frac{e^{-2 \pi i \beta}}{\Gamma(\alpha+\beta)}\left(1+\mathcal{O}\left(\frac{1}{x}\right)\right),
\end{aligned}
$$




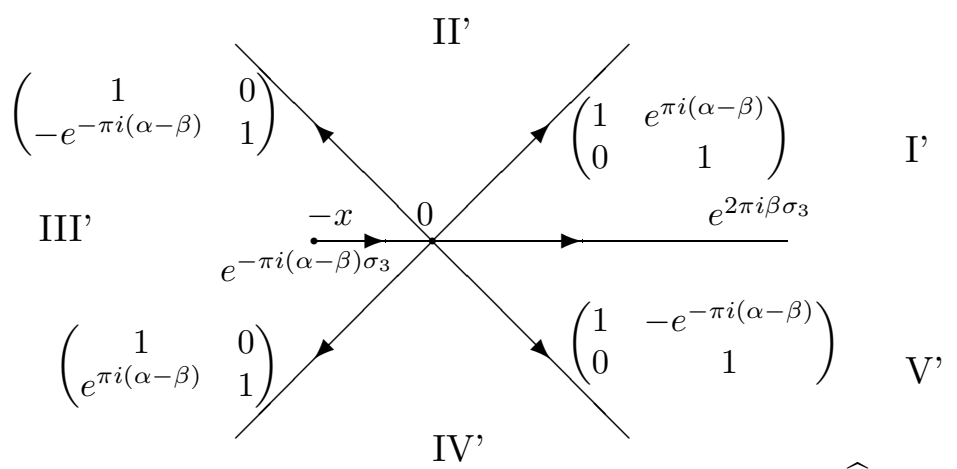

Figure 6: The jump contour and jump matrices for $\widehat{\Psi}$.

$$
\begin{aligned}
\widetilde{C}_{1,21} & =\frac{1}{2 \pi i} \int_{\widehat{\Gamma}_{2}} G(x \zeta ; x)^{-1} d \zeta+\mathcal{O}\left(e^{-x(1-\epsilon)}\right) \\
& =x^{2 \beta} e^{-\frac{x}{2}} \frac{e^{2 \pi i \beta}}{\Gamma(\alpha-\beta)} \psi(1-\alpha+\beta, 2+2 \beta ; x)+\mathcal{O}\left(e^{-x(1-\epsilon)}\right) \\
& =x^{-1+\alpha+\beta} e^{-\frac{x}{2}} \frac{e^{2 \pi i \beta}}{\Gamma(\alpha-\beta)}\left(1+\mathcal{O}\left(\frac{1}{x}\right)\right)
\end{aligned}
$$

and

$$
\widetilde{C}_{1,11}=-\widetilde{C}_{1,22}=\mathcal{O}\left(e^{-\frac{x}{2}(1-\epsilon)}\right),
$$

where $\psi(a, c ; x)$ denotes the confluent hypergeometric function. The last estimate can be improved with the help of the differential identity (4.96) which will be proven in Section 4.3. Indeed, this identity implies that

$$
\frac{d}{d x} \widetilde{C}_{1,11}=\widetilde{C}_{1,12} \widetilde{C}_{1,21}
$$

and hence

$$
\widetilde{C}_{1,11}=x^{-2+2 \alpha} e^{-x} \frac{1}{\Gamma(\alpha-\beta) \Gamma(\alpha+\beta)}\left(1+\mathcal{O}\left(\frac{1}{x}\right)\right) .
$$

Substituting the estimates (4.18), (4.19), and (4.21) into the formula (4.15), we arrive at the following asymptotic equation for the Painlevé function $v(x)$ :

$$
v(x)=x^{-1+2 \alpha} e^{-x} \frac{-1}{\Gamma(\alpha-\beta) \Gamma(\alpha+\beta)}\left(1+\mathcal{O}\left(\frac{1}{x}\right)\right) .
$$

\subsection{Asymptotics for $\Psi$ and $\Phi$ as $x \searrow 0$}

Write $\Psi_{\mathrm{I}}, \ldots, \Psi_{\mathrm{V}}$ for the analytic continuation of $\Psi$ from the indicated in Figure 1 sectors $\mathrm{I}, \ldots, \mathrm{V}$ to $\mathbb{C} \backslash[0,+\infty)$, respectively, and consider the function

$$
\widehat{\Psi}(\lambda ; x):=e^{\frac{x}{2} \sigma_{3}} x^{-\beta \sigma_{3}} \times \begin{cases}\Psi_{\mathrm{I}}\left(\frac{\lambda}{x}+1 ; x\right), & \text { for } \lambda \text { in region I', } \\ \Psi_{\mathrm{II}}\left(\frac{\lambda}{x}+1 ; x\right), & \text { for } \lambda \text { in region II', } \\ \Psi_{\mathrm{III}}\left(\frac{\lambda}{x}+1 ; x\right), & \text { for } \lambda \text { in region III', } \\ \Psi_{\mathrm{IV}}\left(\frac{\lambda}{x}+1 ; x\right), & \text { for } \lambda \text { in region IV', } \\ \Psi_{\mathrm{V}}\left(\frac{\lambda}{x}+1 ; x\right), & \text { for } \lambda \text { in region } V^{\prime}\end{cases}
$$


where the (modified) regions I', .., V' are indicated in Figure 6 , so that $\widehat{\Psi}=\widehat{\Psi}(\lambda ; x, \alpha, \beta)$ has jumps on a contour which is partially shifted compared to the one for $\Psi(\lambda / x+1)$. In particular, the intersection of the contour lines is now at $\lambda=0$ instead of $\lambda=-x / 2$.

From the RH problem for $\Psi$, one easily derives the RH conditions for $\widehat{\Psi}$.

\section{RH problem for $\widehat{\Psi}$}

(a) $\widehat{\Psi}: \mathbb{C} \backslash\left(e^{ \pm \frac{i \pi}{4}} \mathbb{R} \cup \mathbb{R}^{+} \cup[-x, 0]\right) \rightarrow \mathbb{C}^{2 \times 2}$ is analytic.

(b) $\widehat{\Psi}$ has continuous boundary values on $e^{ \pm \frac{i \pi}{4}} \mathbb{R} \cup \mathbb{R}^{+} \cup[-x, 0] \backslash\{-x, 0\}$, and they are related as follows (with the orientation of the contour as in Figure 6),

$$
\begin{array}{lr}
\widehat{\Psi}_{+}(\lambda)=\widehat{\Psi}_{-}(\lambda)\left(\begin{array}{cc}
1 & e^{\pi i(\alpha-\beta)} \\
0 & 1
\end{array}\right), & \text { as } \lambda \in e^{\frac{i \pi}{4}} \mathbb{R}^{+}, \\
\widehat{\Psi}_{+}(\lambda)=\widehat{\Psi}_{-}(\lambda)\left(\begin{array}{cc}
1 & 0 \\
-e^{-\pi i(\alpha-\beta)} & 1
\end{array}\right), & \text { as } \lambda \in e^{\frac{3 i \pi}{4}} \mathbb{R}^{+}, \\
\widehat{\Psi}_{+}(\lambda)=\widehat{\Psi}_{-}(\lambda)\left(\begin{array}{cc}
1 & 0 \\
e^{\pi i(\alpha-\beta)} & 1
\end{array}\right), & \text { as } \lambda \in e^{\frac{5 i \pi}{4}} \mathbb{R}^{+}, \\
\widehat{\Psi}_{+}(\lambda)=\widehat{\Psi}_{-}(\lambda)\left(\begin{array}{cc}
1 & -e^{-\pi i(\alpha-\beta)} \\
0 & 1
\end{array}\right), & \text { as } \lambda \in e^{\frac{7 i \pi}{4}} \mathbb{R}^{+}, \\
\widehat{\Psi}_{+}(\lambda)=\widehat{\Psi}_{-}(\lambda) e^{2 \pi i \beta \sigma_{3}}, & \text { as } \lambda \in \mathbb{R}^{+}, \\
\widehat{\Psi}_{+}(\lambda)=\widehat{\Psi}_{-}(\lambda) e^{-\pi i(\alpha-\beta) \sigma_{3}}, & \text { as } \lambda \in(-x, 0) .
\end{array}
$$

(c) As $\lambda \rightarrow \infty$,

$$
\widehat{\Psi}(\lambda)=\left(I+\mathcal{O}\left(\lambda^{-1}\right)\right) \lambda^{-\beta \sigma_{3}} e^{-\frac{1}{2} \lambda \sigma_{3}} .
$$

(d0) As $\lambda \rightarrow-x$,

$$
\widehat{\Psi}(\lambda)=\mathcal{O}\left(\begin{array}{ll}
|\lambda+x|^{\frac{\alpha-\beta}{2}} & |\lambda+x|^{-\frac{\alpha-\beta}{2}} \\
|\lambda+x|^{\frac{\alpha-\beta}{2}} & |\lambda+x|^{-\frac{\alpha-\beta}{2}}
\end{array}\right) .
$$

(d1) As $\lambda \rightarrow 0$ in sectors I' and V',

$$
\widehat{\Psi}(\lambda)=\mathcal{O}\left(\begin{array}{ll}
|\lambda|^{-\frac{\alpha+\beta}{2}} & |\lambda|^{\frac{\alpha+\beta}{2}} \\
|\lambda|^{-\frac{\alpha+\beta}{2}} & |\lambda|^{\frac{\alpha+\beta}{2}}
\end{array}\right) .
$$

As $\lambda \rightarrow 0$ in the other sectors, the behaviour of $\widehat{\Psi}$ is obtained by applying the jump conditions to (4.32). $\widehat{\Psi}(\lambda)=\mathcal{O}\left(\lambda^{-\frac{|\alpha+\beta|}{2}}\right)$ always holds.

For small values of $x$, we will now construct a global parametrix and a local parametrix near 0 for $\widehat{\Psi}$ and match them on the boundary of an $\varepsilon$-neighborhood of $\lambda=0$. These constructions will lead to the uniform asymptotics for $\widehat{\Psi}$ and $\Psi$ as $x \searrow 0$.

\subsubsection{Construction of the global parametrix}

Consider a fixed $\varepsilon$-neighborhood $U_{\varepsilon}$ of $\lambda=0$ containing, in particular, the $[-x, 0]$ part of the contour. Outside of this neighborhood, we expect to model $\widehat{\Psi}$ by the global parametrix $M=M(\lambda ; \alpha, \beta)$ independent of $x$ and solving the following $\mathrm{RH}$ problem. 


\section{RH problem for $M$}

(a) $M: \mathbb{C} \backslash\left(e^{ \pm \frac{\pi i}{4}} \mathbb{R} \cup \mathbb{R}^{+}\right) \rightarrow \mathbb{C}^{2 \times 2}$ is analytic,

(b) $M$ has continuous boundary values on $e^{ \pm \frac{\pi i}{4}} \mathbb{R} \cup \mathbb{R}^{+} \backslash\{0\}$ related by the conditions

$$
\begin{array}{lr}
M_{+}(\lambda)=M_{-}(\lambda)\left(\begin{array}{cc}
1 & e^{\pi i(\alpha-\beta)} \\
0 & 1
\end{array}\right), & \text { as } \lambda \in e^{\frac{i \pi}{4}} \mathbb{R}^{+}, \\
M_{+}(\lambda)=M_{-}(\lambda)\left(\begin{array}{cc}
1 & 0 \\
-e^{-\pi i(\alpha-\beta)} & 1
\end{array}\right), & \text { as } \lambda \in e^{\frac{3 i \pi}{4}} \mathbb{R}^{+}, \\
M_{+}(\lambda)=M_{-}(\lambda)\left(\begin{array}{cr}
1 & 0 \\
e^{\pi i(\alpha-\beta)} & 1
\end{array}\right), & \text { as } \lambda \in e^{\frac{5 i \pi}{4}} \mathbb{R}^{+}, \\
M_{+}(\lambda)=M_{-}(\lambda)\left(\begin{array}{cc}
1 & -e^{-\pi i(\alpha-\beta)} \\
0 & 1
\end{array}\right), & \text { as } \lambda \in e^{\frac{7 i \pi}{4}} \mathbb{R}^{+}, \\
M_{+}(\lambda)=M_{-}(\lambda) e^{2 \pi i \beta \sigma_{3}}, & \text { as } \lambda \in \mathbb{R}^{+} .
\end{array}
$$

(c) We have

$$
M(\lambda)=\left(I+\mathcal{O}\left(\lambda^{-1}\right)\right) \lambda^{-\beta \sigma_{3}} e^{-\frac{1}{2} \lambda \sigma_{3}}, \quad \text { as } \lambda \rightarrow \infty .
$$

We can solve this RH problem explicitly in terms of the confluent hypergeometric function. Inspired by the constructions of [23, 11, we define

$$
\begin{aligned}
& H(\lambda):=\left(\begin{array}{cc}
e^{-i \pi(2 \beta+\alpha)} & 0 \\
0 & e^{i \pi(\beta+2 \alpha)}
\end{array}\right) e^{-\frac{i \pi}{2} \alpha \sigma_{3}}\left(\begin{array}{c}
\lambda^{\alpha} \psi(\alpha+\beta, 1+2 \alpha, \lambda) e^{i \pi(2 \beta+\alpha)} \\
\lambda^{-\alpha} \psi(1-\alpha+\beta, 1-2 \alpha, \lambda) e^{i \pi(\beta-3 \alpha)} \frac{\Gamma(1+\alpha+\beta)}{\Gamma(\alpha-\beta)}
\end{array}\right.
\end{aligned}
$$

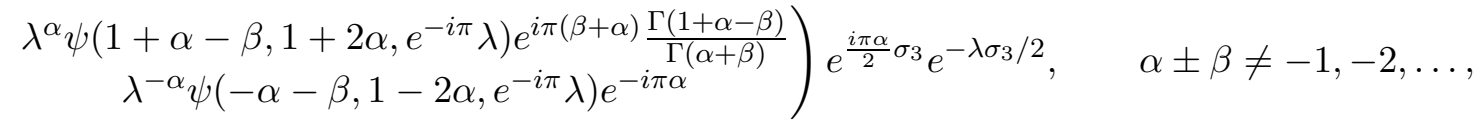

where $\psi(a, b, x)$ is the confluent hypergeometric function, and $\Gamma(x)$ is Euler's $\Gamma$-function. Furthermore let

$$
\begin{aligned}
& M(\lambda)=M_{1}(\lambda):=H(\lambda)\left(\begin{array}{cc}
1 & -e^{\pi i(\alpha-\beta)} \\
0 & 1
\end{array}\right), \quad \quad \text { for } 0<\arg \lambda<\frac{\pi}{4}, \\
& M(\lambda)=M_{2}(\lambda):=H(\lambda), \quad \text { for } \frac{\pi}{4}<\arg \lambda<\frac{3 \pi}{4}, \\
& M(\lambda)=M_{3}(\lambda):=H(\lambda)\left(\begin{array}{cc}
1 & 0 \\
-e^{-\pi i(\alpha-\beta)} & 1
\end{array}\right), \quad \quad \text { for } \frac{3 \pi}{4}<\arg \lambda<\frac{5 \pi}{4}, \\
& M(\lambda)=M_{4}(\lambda):=H(\lambda)\left(\begin{array}{cc}
1 & 0 \\
2 i \sin \pi(\alpha-\beta) & 1
\end{array}\right), \quad \text { for } \frac{5 \pi}{4}<\arg \lambda<\frac{7 \pi}{4}, \\
& M(\lambda)=M_{5}(\lambda):=H(\lambda) \\
& \times\left(\begin{array}{cc}
1 & -e^{-\pi i(\alpha-\beta)} \\
2 i \sin \pi(\alpha-\beta) & -2 i e^{-\pi i(\alpha-\beta)} \sin \pi(\alpha-\beta)+1
\end{array}\right), \quad \text { for } \frac{7 \pi}{4}<\arg \lambda<2 \pi .
\end{aligned}
$$

Using known properties of the confluent hypergeometric function, one verifies as in [1] that $M$ satisfies the prescribed $\mathrm{RH}$ conditions. 
In order to match $M$ later on with the local parametrix near zero that we construct in the next section, we will now need to rewrite $M$ in the form in which the structure of its singularity at $\lambda=0$ becomes more apparent.

Recall the following properties of the confluent hypergeometric function (see, e.g., [5]):

$$
\begin{aligned}
& \frac{\phi(a, c, z)}{\Gamma(c)}=\frac{e^{i \pi a}}{\Gamma(c-a)} \psi(a, c, z)+\frac{e^{-i \pi(c-a)}}{\Gamma(a)} \psi\left(c-a, c, e^{-i \pi} z\right) e^{z} \\
& \psi(a, c, z)=z^{1-c} \psi(a-c+1,2-c, z) \\
& \psi(a, c, z)=\frac{\Gamma(1-c)}{\Gamma(a-c+1)} \varphi(a, c, z)+\frac{\Gamma(c-1)}{\Gamma(a)} z^{1-c} \varphi(a-c+1,2-c, z), \\
& \varphi(a, c, z)=e^{z} \varphi(c-a, c,-z)
\end{aligned}
$$

where

$$
\varphi(a, c ; z)=1+\sum_{n=1}^{\infty} \frac{a(a+1) \cdots(a+n-1)}{c(c+1) \cdots(c+n-1)} \frac{z^{n}}{n !}, \quad c \neq 0,-1,-2, \ldots
$$

is an entire function. Let us focus on the region III'. Assume first that $2 \alpha \neq 0,1,2, \ldots$ (noninteger $2 \alpha$ combined with our general condition $\operatorname{Re} \alpha>-1 / 2$ ). Using the properties (4.40) and (4.41) for simplification of the first column of $M_{3}$, and (4.42), (4.43) for the second, we easily obtain

$$
M_{3}(\lambda)=E(\lambda) \lambda^{\alpha \sigma_{3}} G_{3}, \quad 2 \alpha \neq 0,1, \ldots, \quad \alpha \pm \beta \neq-1,-2, \ldots,
$$

with the branch of $\lambda^{ \pm \alpha}$ chosen with $0<\arg \lambda<2 \pi$. Here

$$
E(\lambda)=e^{-\lambda / 2}\left(\begin{array}{r}
e^{-i \pi(\alpha+\beta)} \frac{\Gamma(1+\alpha-\beta)}{\Gamma(1+2 \alpha)} \varphi(\alpha+\beta, 1+2 \alpha, \lambda) \\
-e^{-i \pi(\alpha-\beta)} \frac{\Gamma(1+\alpha+\beta)}{\Gamma(1+2 \alpha)} \varphi(1+\alpha+\beta, 1+2 \alpha, \lambda) \\
e^{i \pi(\alpha-\beta)} \frac{\Gamma(2 \alpha)}{\Gamma(\alpha+\beta)} \varphi(-\alpha+\beta, 1-2 \alpha, \lambda) \\
e^{i \pi(\alpha+\beta)} \frac{\Gamma(2 \alpha)}{\Gamma(\alpha-\beta)} \varphi(1-\alpha+\beta, 1-2 \alpha, \lambda)
\end{array}\right)
$$

is entire, and $G_{3}$ is the constant matrix

$$
G_{3}=\left(\begin{array}{cc}
1 & c_{1} \\
0 & 1
\end{array}\right), \quad c_{1}=-\frac{\sin \pi(\alpha+\beta)}{\sin 2 \pi \alpha} .
$$

Applying to (4.45) the jump conditions we readily obtain the general formulas

$$
M_{j}(\lambda)=E(\lambda) \lambda^{\alpha \sigma_{3}} G_{j}, \quad j=1, \ldots, 5, \quad 2 \alpha \neq 0,1, \ldots, \quad \alpha \pm \beta \neq-1,-2, \ldots,
$$

with appropriate constant matrices $G_{j}$. In particular, $G_{3}$ is given by (4.47) and

$$
G_{1}=\left(\begin{array}{cc}
1+c_{1} e^{-i \pi(\alpha-\beta)} & -e^{i \pi(\alpha-\beta)} \\
e^{-i \pi(\alpha-\beta)} & 0
\end{array}\right)
$$

Consider now the case when $2 \alpha$ is an integer. In this case, the calculations for the first column of $M_{3}$ remain the same, whereas for the second column (note that (4.42) 
does not hold now) we use the known logarithmic formulas for the $\psi$-function. We then obtain

$$
\begin{array}{lr}
M_{j}(\lambda)=\widetilde{E}(\lambda) \lambda^{\alpha \sigma_{3}}\left(\begin{array}{cc}
1 & \gamma(\lambda) \\
0 & 1
\end{array}\right) \widetilde{G}_{j}, & j=1, \ldots, 5, \\
\gamma(\lambda)=\frac{(-1)^{2 \alpha+1}}{\pi} \sin \pi(\alpha+\beta) \ln \left(\lambda e^{-i \pi}\right), & \text { if } \quad 2 \alpha=0,1, \ldots,
\end{array}
$$

where $\widetilde{E}(\lambda)$ is analytic at zero, and $\widetilde{G}_{j}$ are constant matrices. In particular, $\widetilde{G}_{3}=I$.

\subsubsection{Construction of the local parametrix near 0}

We construct a local parametrix $P$ in $U_{\varepsilon}$ in such a way that it has the singularities and jumps of $\widehat{\Psi}$ and matches with $M$ to the main order in $x$ on the boundary. We have to consider the cases $2 \alpha \notin \mathbb{Z}$ and $2 \alpha \in \mathbb{Z}$ separately because of the different behavior of $M$ at $\partial U_{\varepsilon}$. As examination of the final formulas show (see (4.61), (4.64), (4.71), and (4.72) below), our constructions in this section will be valid for all $\alpha, \beta$ such that $\alpha \pm \beta \neq-1,-2, \ldots$ This is exactly the restriction on $M$ in the previous section. Some preliminary expressions, however, are valid under stronger conditions $\alpha \pm \beta \notin \mathbb{Z}$ (cf. (4.56) ). We do not mention these conditions as they disappear in the final formulas, namely: the singularities in $\alpha, \beta$ of $J$ and $\widetilde{J}$ defined below cancel with the zeros of $c_{0}$, $c_{2}$.

We first deal with the case when $2 \alpha \neq 0,1,2, \ldots$. Since we have a problem with 2 singular points with power-law behaviour at $-x$ and 0 and require a power-law behaviour at the boundary $\partial U_{\varepsilon}$ (see (4.48)) we expect from the general principles (e.g., [16]) a parametrix in terms of the hypergeometric function. Indeed such a parametrix was found by Jimbo [25] in the generic case of Painlevé $\mathrm{V}$ equation. Instead of trying to specialize it to our situation, we provide a direct construction below. For $2 \alpha \notin \mathbb{Z}$, define $P=P(\lambda ; x, \alpha, \beta)$ in $U_{\varepsilon}$ by the expressions

$$
P(\lambda)=P_{j}(\lambda), \quad \text { with } j=1 \text { for } \lambda \text { in sector I', } j=2 \text { in sector II', and so on, }
$$

where

$$
P_{j}(\lambda)=E(\lambda)\left(\begin{array}{cc}
1 & c_{0} J(\lambda ; x, \alpha, \beta) \\
0 & 1
\end{array}\right)(\lambda+x)^{\frac{\alpha-\beta}{2} \sigma_{3}} \lambda^{\frac{\alpha+\beta}{2} \sigma_{3}} G_{j}
$$

with the argument of the roots between 0 and $2 \pi$. Here $E$ is given by (4.46), $G_{j}$ are as in (4.48),

$$
\begin{gathered}
J(\lambda ; x, \alpha, \beta)=\frac{1}{\pi} \frac{x^{1+2 \alpha}}{-\lambda} \frac{\Gamma(1+\alpha+\beta) \Gamma(1+\alpha-\beta)}{\Gamma(2+2 \alpha)} F\left(1,1+\alpha+\beta, 2+2 \alpha, e^{i \pi} x / \lambda\right), \\
c_{0}=-e^{2 \pi i \alpha} \frac{\sin \pi(\alpha+\beta) \sin \pi(\alpha-\beta)}{\sin 2 \pi \alpha},
\end{gathered}
$$

and $F(a, b, c, z)$ is the hypergeometric function of $z$ with parameters $a, b, c$. The argument of $z$ is chosen between 0 and $2 \pi$. For $c \neq 0,-1,-2, \ldots$, this function is represented by the standard series

$$
F(a, b, c, z)=1+\sum_{n=1}^{\infty} \frac{a(a+1) \cdots(a+n-1) b(b+1) \cdots(b+n-1)}{c(c+1) \cdots(c+n-1)} \frac{z^{n}}{n !}
$$


converging in the disk $|z| \leq r<1$ of any radius $r<1$, and is extended to the analytic function in the plane with a cut $[1,+\infty)$. Therefore, the function $F\left(a, b, c, e^{i \pi} x / \lambda\right)$, in particular the one in (4.54), is analytic in $\lambda$-plane outside $[-x, 0]$. We will now find the jump of $J(\lambda)$ on $[-x, 0]$ and the structure of its singularities at $-x$ and 0 . First, using the transformation of the hypergeometric functions from the one with the argument $z$ to those with the argument $1 / z$, we can write:

$$
\begin{aligned}
& F(1,1+\alpha+\beta, 2+2 \alpha, z)=-\frac{\pi}{\sin \pi(\alpha+\beta)} \frac{\Gamma(2+2 \alpha)}{\Gamma(1+\alpha+\beta) \Gamma(1+\alpha-\beta)} \\
& \times\left(e^{i \pi} z^{-1}\right)^{1+\alpha+\beta} F(1+\alpha+\beta,-\alpha+\beta, 1+\alpha+\beta, 1 / z) \\
& +\frac{1+2 \alpha}{\alpha+\beta} e^{i \pi} z^{-1} F(1,-2 \alpha, 1-\alpha-\beta, 1 / z) .
\end{aligned}
$$

Since the function

$$
F(1+\alpha+\beta,-\alpha+\beta, 1+\alpha+\beta, 1 / z)=\left(1-\frac{1}{z}\right)^{\alpha-\beta},
$$

and $F(1,-2 \alpha, 1-\alpha-\beta, 1 / z)$ are analytic outside $[0,1]$, we have on $(1,+\infty)$ :

$$
\begin{aligned}
& F(1,1+\alpha+\beta, 2+2 \alpha, z)_{+}-F(1,1+\alpha+\beta, 2+2 \alpha, z)_{-} \\
& =\frac{2 \pi i \Gamma(2+2 \alpha)}{\Gamma(1+\alpha+\beta) \Gamma(1+\alpha-\beta)}|z|^{-1-\alpha-\beta}\left|1-\frac{1}{z}\right|^{\alpha-\beta}, \quad z \in(1,+\infty) .
\end{aligned}
$$

Setting here $z=e^{i \pi} x / \lambda$ and using (4.54), we obtain

$$
J_{+}(\lambda)=J_{-}(\lambda)+2 i|\lambda|^{\alpha+\beta}|\lambda+x|^{\alpha-\beta}, \quad \lambda \in(-x, 0) .
$$

By (4.48) we can write

$$
P(\lambda)=E(\lambda)\left(\begin{array}{cc}
1 & c_{0} J(\lambda) \\
0 & 1
\end{array}\right)(\lambda+x)^{\frac{\alpha-\beta}{2} \sigma_{3}} \lambda^{-\frac{\alpha-\beta}{2} \sigma_{3}} E(\lambda)^{-1} M(\lambda) .
$$

Since the product of the factors to the left of $M$ is analytic in $\mathbb{C} \backslash[-x, 0]$, the expression (4.58) implies directly that $P$ satisfies the jump conditions for $M$, see (4.33)-(4.37), except on $(-x, 0)$ where $M$ is analytic but $P$ is not. For $\lambda \in(-x, 0)$, it is convenient to use (4.53), (4.47), and (4.57) to verify that

$$
P_{+}(\lambda)=P_{-}(\lambda) e^{-\pi i(\alpha-\beta) \sigma_{3}} .
$$

This is only true if $c_{0}$ is given by (4.55). We have thus constructed $P$ in such a way that it has exactly the jump conditions for $\widehat{\Psi}$.

From the fact that $P$ and $\widehat{\Psi}$ have the same jumps, it follows that $\widehat{\Psi} P^{-1}$ is analytic in $U_{\varepsilon}$ except possibly at the points $-x$ and 0 . Let us investigate the behavior of $\widehat{\Psi} P^{-1}$ near these points in some detail. Recall that by Proposition 4.1$](\zeta) \zeta^{-\frac{\alpha-\beta}{2} \sigma_{3}}(\zeta-1)^{\frac{\alpha+\beta}{2} \sigma_{3}}$ is analytic at 0 and 1 , which implies by (4.23) that $\widehat{\Psi}_{0}(\lambda):=\widehat{\Psi}(\lambda)(\lambda+x)^{-\frac{\alpha-\beta}{2} \sigma_{3}} \lambda^{\frac{\alpha+\beta}{2} \sigma_{3}}$ is analytic at $-x$ and 0 , where at $-x$ the variable $\lambda$ is in the region $I I I^{\prime}$, while at 0 , the variable $\lambda$ is in the region $I^{\prime}$ or $V^{\prime}$ (for other regions at 0 , the appropriate jump conditions should be applied to $\widehat{\Psi}(\lambda)$ ). Consider first $\lambda$ close to 0 . By (4.53), we have

$$
\begin{aligned}
\widehat{\Psi}(\lambda) P(\lambda)^{-1}=\widehat{\Psi}_{0}(\lambda)(\lambda+ & x)^{\frac{\alpha-\beta}{2} \sigma_{3}} \lambda^{-\frac{\alpha+\beta}{2} \sigma_{3}} G_{1}^{-1}(\lambda+x)^{-\frac{\alpha-\beta}{2} \sigma_{3}} \lambda^{-\frac{\alpha+\beta}{2} \sigma_{3}} \\
& \times\left(\begin{array}{cc}
1 & -c_{0} J(\lambda ; x, \alpha, \beta) \\
0 & 1
\end{array}\right) E(\lambda)^{-1}, \quad \lambda \in I^{\prime} .
\end{aligned}
$$


Substituting (4.56) with $z=e^{i \pi} x / \lambda$ into (4.54), we obtain the following representation

$$
J(\lambda ; x, \alpha, \beta)=\frac{\lambda^{\alpha+\beta}(\lambda+x)^{\alpha-\beta}}{\sin \pi(\alpha+\beta)}-x^{2 \alpha} \frac{\Gamma(\alpha+\beta) \Gamma(1+\alpha-\beta)}{\pi \Gamma(1+2 \alpha)} F(1,-2 \alpha, 1-\alpha-\beta,-\lambda / x) .
$$

Therefore,

$$
\begin{aligned}
& c_{0} J(\lambda ; x, \alpha, \beta)=-e^{2 \pi i \alpha} \frac{\sin \pi(\alpha-\beta)}{\sin 2 \pi \alpha} \lambda^{\alpha+\beta}(\lambda+x)^{\alpha-\beta}+F_{0}(\lambda), \\
& F_{0}(\lambda)=-x^{2 \alpha} e^{2 \pi i \alpha} \frac{\sin \pi(\alpha-\beta)}{\pi} \frac{\Gamma(1+\alpha-\beta) \Gamma(-2 \alpha)}{\Gamma(1-\alpha-\beta)} F(1,-2 \alpha, 1-\alpha-\beta,-\lambda / x) .
\end{aligned}
$$

Since $F_{0}(\lambda)$ is analytic close to $\lambda=0$, this representation gives an explicit expression for the singularity of $J(\lambda)$ at 0 . (Note that the arguments of $\lambda, \lambda+x$ here are between $-\pi$ and $\pi$ as they originate from the hypergeometric function above, whereas the arguments

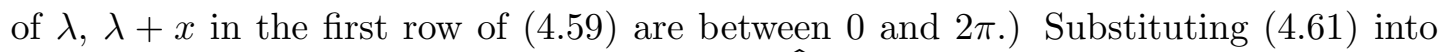
(4.59) we see that the singularity cancels, and $\widehat{\Psi}(\lambda) P(\lambda)^{-1}$ is analytic at $\lambda=0$.

In order to analyze the singularity of $J$ at $\lambda=-x$, apply first the transformation of the hypergeometric function between arguments $z$ and $1-z$ to (4.56) with $z=e^{i \pi} x / \lambda$. We then obtain:

$$
\begin{aligned}
& F(1,1+\alpha+\beta, 2+2 \alpha,-x / \lambda)= \\
& \frac{\pi e^{-2 \pi i \alpha}}{\sin \pi(\alpha-\beta)} \frac{\Gamma(2+2 \alpha)}{\Gamma(1+\alpha+\beta) \Gamma(1+\alpha-\beta)}\left(\frac{\lambda}{x}\right)^{1+\alpha+\beta}\left(1+\frac{\lambda}{x}\right)^{\alpha-\beta} \\
& -\frac{1+2 \alpha}{\alpha-\beta} \frac{\lambda}{x} F(1,-2 \alpha, 1-\alpha+\beta, 1+\lambda / x),
\end{aligned}
$$

and therefore by (4.54),

$$
\begin{aligned}
& J(\lambda ; x, \alpha, \beta)=-\frac{e^{-2 \pi i \alpha} \lambda^{\alpha+\beta}(\lambda+x)^{\alpha-\beta}}{\sin \pi(\alpha-\beta)} \\
& +x^{2 \alpha} \frac{\Gamma(\alpha-\beta) \Gamma(1+\alpha+\beta)}{\pi \Gamma(1+2 \alpha)} F(1,-2 \alpha, 1-\alpha+\beta, 1+\lambda / x) .
\end{aligned}
$$

Using (4.55), we obtain

$$
\begin{aligned}
& c_{0} J(\lambda ; x, \alpha, \beta)=\frac{\sin \pi(\alpha+\beta)}{\sin 2 \pi \alpha} \lambda^{\alpha+\beta}(\lambda+x)^{\alpha-\beta}+F_{1}(\lambda), \\
& F_{1}(\lambda)=x^{2 \alpha} e^{2 \pi i \alpha} \frac{\sin \pi(\alpha+\beta)}{\pi} \frac{\Gamma(1+\alpha+\beta) \Gamma(-2 \alpha)}{\Gamma(1-\alpha+\beta)} F(1,-2 \alpha, 1-\alpha+\beta, 1+\lambda / x) .
\end{aligned}
$$

This representation explicitly displays the singularity at $-x$ because $F_{1}(\lambda)$ is analytic near $\lambda=-x$. In the same way as for $\lambda=0$, we now obtain that $\widehat{\Psi}(\lambda) P(\lambda)^{-1}$ is analytic at $\lambda=-x$ as well.

For $\lambda$ at a fixed distance away of the origin, say for $\lambda \in \partial U_{\varepsilon}$, it follows from (4.58) that

$$
P(\lambda ; x) M(\lambda)^{-1}=I+\mathcal{O}(x)+\mathcal{O}\left(x^{1+2 \alpha}\right), \quad \text { as } x \searrow 0 .
$$


We now consider the case $2 \alpha \in \mathbb{Z}$. We again set

$$
P(\lambda)=P_{j}(\lambda), \quad \text { with } j=1 \text { for } \lambda \text { in sector I', } j=2 \text { in sector II', and so on, (4.66) }
$$

but now with

$$
P_{j}(\lambda)=\widetilde{E}(\lambda)\left(\begin{array}{cc}
1 & c_{2} \widetilde{J}(\lambda) \\
0 & 1
\end{array}\right)(\lambda+x)^{\frac{\alpha-\beta}{2} \sigma_{3}} \lambda^{\frac{\alpha+\beta}{2} \sigma_{3}}\left(\begin{array}{cc}
1 & \gamma(\lambda) \\
0 & 1
\end{array}\right) \widetilde{G}_{j},
$$

where

$$
\begin{aligned}
& c_{2}=-\frac{1}{\pi} \sin \pi(\alpha+\beta) \sin \pi(\alpha-\beta), \\
& \widetilde{J}(\lambda ; x, \alpha, \beta)=\frac{1}{2}\left(\frac{\partial}{\partial \alpha}+\frac{\partial}{\partial \beta}\right) J(\lambda ; x, \alpha, \beta),
\end{aligned}
$$

with $J(\lambda)$ given by (4.54).

Note that

$$
\widetilde{J}_{+}(\lambda)=\widetilde{J}_{-}(\lambda)+2 i|\lambda|^{\alpha+\beta}|\lambda+x|^{\alpha-\beta} \ln |\lambda|, \quad \lambda \in(-x, 0) .
$$

A similar derivation to the one above shows that $P$ again satisfies the same jump conditions as the ones for $\widehat{\Psi}$. To analyze the structure of the singularities of $P(\lambda)$ at 0 and at $-x$, we need, as above, to find suitable expressions for $c_{2} \widetilde{J}(\lambda)$ at these points. Applying the differential operator $\frac{1}{2}(\partial / \partial \alpha+\partial / \partial \beta)$ to (4.60) (it is convenient to write $\lambda^{\alpha+\beta}=e^{i \pi(\alpha+\beta)}\left(e^{-i \pi} \lambda\right)^{\alpha+\beta}$ first) and then taking $2 \alpha \in \mathbb{Z}$ and using the fact that

$$
\frac{\sin \pi(\alpha-\beta)}{\sin \pi(\alpha+\beta)}=(-1)^{2 \alpha+1}, \quad 2 \alpha \in \mathbb{Z}, \quad \beta \notin \mathbb{Z},
$$

we obtain

$$
\begin{aligned}
& c_{2} \widetilde{J}(\lambda ; x, \alpha, \beta)=\left[(-1)^{2 \alpha+1}\left(\lambda e^{-i \pi}\right)^{\alpha+\beta}-\frac{1}{\pi} \sin \pi(\alpha-\beta) \lambda^{\alpha+\beta} \ln \left(\lambda e^{-i \pi}\right)\right](\lambda+x)^{\alpha-\beta}+\widetilde{F}_{0}(\lambda) \\
& \widetilde{F}_{0}(\lambda)=-\frac{c_{2}}{2}\left(\frac{\partial}{\partial \alpha}+\frac{\partial}{\partial \beta}\right)\left[x^{2 \alpha} \frac{\Gamma(\alpha+\beta) \Gamma(1+\alpha-\beta)}{\pi \Gamma(1+2 \alpha)} F(1,-2 \alpha, 1-\alpha-\beta,-\lambda / x)\right] .
\end{aligned}
$$

As above, this expression can be used to show that $\widehat{\Psi}(\lambda) P(\lambda)^{-1}$ has no singularity at $\lambda=0$. To analyze a neighborhood of $\lambda=-x$, note that the first term on the r.h.s. of (4.63) can be written as

$$
-\frac{e^{-\pi i(\alpha-\beta)}}{\sin \pi(\alpha-\beta)}\left(e^{-i \pi} \lambda\right)^{\alpha+\beta}(\lambda+x)^{\alpha-\beta},
$$

and application of the operator $\frac{1}{2}(\partial / \partial \alpha+\partial / \partial \beta)$ to the fraction in the above formula gives zero by antisymmetry in $\alpha$ and $\beta$. We finally obtain from (4.63)

$$
\begin{aligned}
& c_{2} \widetilde{J}(\lambda ; x, \alpha, \beta)=\frac{1}{\pi} \sin \pi(\alpha+\beta) e^{-2 \pi \alpha} \lambda^{\alpha+\beta}(\lambda+x)^{\alpha-\beta} \ln \left(\lambda e^{-i \pi}\right)+\widetilde{F}_{1}(\lambda) \\
& \widetilde{F}_{1}(\lambda)=\frac{c_{2}}{2}\left(\frac{\partial}{\partial \alpha}+\frac{\partial}{\partial \beta}\right)\left[x^{2 \alpha} \frac{\Gamma(\alpha-\beta) \Gamma(1+\alpha+\beta)}{\pi \Gamma(1+2 \alpha)} F(1,-2 \alpha, 1-\alpha+\beta, 1+\lambda / x)\right],
\end{aligned}
$$


which can be used to see that $\widehat{\Psi}(\lambda) P(\lambda)^{-1}$ has no singularity at $\lambda=-x$.

It is easy to see that

$$
P(\lambda ; x) M(\lambda)^{-1}=I+\mathcal{O}(x \ln x), \quad \text { for } \lambda \in \partial U,
$$

as $x \searrow 0$.

Note that using an integral representation for the hypergeometric function, we can, if $\operatorname{Re}(\alpha \pm \beta)>-1$, represent $J(\lambda)$ and $\widetilde{J}(\lambda)$ in the following form, which makes the jump conditions (4.57) and (4.70) obvious:

$$
\begin{aligned}
& J(\lambda)=\frac{1}{\pi} \int_{-x}^{0} \frac{|\xi+x|^{\alpha-\beta}|\xi|^{\alpha+\beta}}{\xi-\lambda} d \xi, \quad \widetilde{J}(\lambda)=\frac{1}{\pi} \int_{-x}^{0} \frac{|\xi+x|^{\alpha-\beta}|\xi|^{\alpha+\beta} \ln |\xi|}{\xi-\lambda} d \xi \\
& \operatorname{Re}(\alpha \pm \beta)>-1 .
\end{aligned}
$$

Now define

$$
R(\lambda)= \begin{cases}\widehat{\Psi}(\lambda) M(\lambda)^{-1}, & \text { for } \lambda \in \mathbb{C} \backslash U_{\varepsilon}, \\ \widehat{\Psi}(\lambda) P(\lambda)^{-1}, & \text { for } \lambda \in U_{\varepsilon} .\end{cases}
$$

This function satisfies the following problem.

\section{RH problem for $R$}

(a) $R$ is analytic in $\mathbb{C} \backslash \partial U_{\varepsilon}$.

(b) The jump condition for $R$ is

$$
R_{+}(\lambda)=R_{-}(\lambda)(I+e(\lambda)), \quad \text { for } \lambda \in \partial U, \text { with } e(\lambda)=o(1) \text { as } x \searrow 0 .
$$

(c) $R(\lambda)=I+\mathcal{O}\left(\lambda^{-1}\right)$ as $\lambda \rightarrow \infty$.

For $x$ sufficiently small, this is a small-norm $\mathrm{RH}$ problem, and it follows that the $\mathrm{RH}$ problem for $R$ is solvable, say for $0<x<\delta$. From the invertible transformations $\Psi \mapsto \widehat{\Psi} \mapsto R$ and $\Psi \mapsto \Phi$, it follows that the RH problems for $\Psi$ and $\Phi$ are solvable as well for $0<x<\delta$. We also have $R(\lambda)=I+o(1)$ uniformly for $\lambda \in \mathbb{C} \backslash \partial U$ as $x \searrow 0$. In particular this holds at infinity, which means that

$$
R(\lambda)=I+\mathcal{O}\left(\frac{1}{\lambda}\right), \quad \text { as } \lambda \rightarrow \infty
$$

uniformly for small $x$. Tracing back the transformations $\Psi \mapsto \widehat{\Psi} \mapsto R$ and $\Psi \mapsto \Phi$, we can conclude that

$$
\Phi(\lambda ; x)=I+\mathcal{O}\left(\lambda^{-1}\right), \quad \text { as } \lambda \rightarrow \infty, \text { uniformly for } 0<x<\delta .
$$

Moreover, using (4.61) we obtain for $2 \alpha \notin \mathbb{Z}$

$$
\Phi\left(\frac{x}{2} ; x\right) \sigma_{3} \Phi\left(\frac{x}{2} ; x\right)^{-1}=e^{-x \sigma_{3} / 4} R(0) E(0)\left(\begin{array}{cc}
-1 & 2 F_{0}(0) \\
0 & 1
\end{array}\right) E(0)^{-1} R(0)^{-1} e^{x \sigma_{3} / 4},
$$


where $F_{0}(\lambda)$ is defined in (4.61). Similarly,

$$
\begin{aligned}
& \Phi\left(-\frac{x}{2} ; x\right) \sigma_{3} \Phi\left(-\frac{x}{2} ; x\right)^{-1}= \\
& e^{-x \sigma_{3} / 4} R(-x) E(-x)\left(\begin{array}{cc}
1 & -2 F_{1}(-x) \\
0 & -1
\end{array}\right) E(-x)^{-1} R(-x)^{-1} e^{x \sigma_{3} / 4},
\end{aligned}
$$

where $F_{1}(\lambda)$ is defined in (4.64).

Therefore, we have

$$
\Phi\left( \pm \frac{x}{2} ; x\right) \sigma_{3} \Phi\left( \pm \frac{x}{2} ; x\right)^{-1}=\mathcal{O}(1)+\mathcal{O}\left(x^{2 \alpha}\right), \quad x \searrow 0, \quad 2 \alpha \notin \mathbb{Z} .
$$

Similarly, by (4.67), 44.71), 4.72),

$$
\Phi\left( \pm \frac{x}{2} ; x\right) \sigma_{3} \Phi\left( \pm \frac{x}{2} ; x\right)^{-1}=\mathcal{O}(1)+\mathcal{O}\left(x^{2 \alpha}\right)+\mathcal{O}\left(x^{2 \alpha} \ln x\right), \quad x \searrow 0, \quad 2 \alpha \in \mathbb{Z}
$$

We will now estimate $w(x)$ given by (3.45) as $x \searrow 0$. First, using the connection (3.9) between $\Phi$ and $\Psi$, we obtain

$$
\left(\Phi^{-1} \Phi_{\lambda}^{\prime}\right)_{j j}=\left(\Psi^{-1} \Psi_{\lambda}^{\prime}\right)_{j j}-\frac{(-1)^{j}}{2}\left(\frac{\alpha+\beta}{\lambda-x / 2}-\frac{\alpha-\beta}{\lambda+x / 2}+1\right), \quad j=1,2 .
$$

Expressing $\Psi^{-1} \Psi_{\lambda}^{\prime}=\widehat{\Psi}^{-1} \widehat{\Psi}_{\lambda}^{\prime}$, and using (4.75), and the formulas for $P(\lambda)$, we obtain after straightforward calculations that

$$
\begin{aligned}
& \left(\Phi^{-1} \Phi_{\lambda}^{\prime}\right)_{11}(x / 2)=-\frac{\alpha-\beta}{x}+\left(d_{1}+d_{2} x^{2 \alpha}\right)(1+\mathcal{O}(x)), \quad d_{1}=\frac{\alpha-\beta}{2 \alpha}, \\
& d_{2}=\frac{\alpha-\beta}{1+2 \alpha} \frac{\Gamma(1+\alpha+\beta) \Gamma(1+\alpha-\beta)}{\Gamma(1-\alpha+\beta) \Gamma(1-\alpha-\beta)} \frac{\Gamma(-2 \alpha)}{\Gamma(1+2 \alpha)^{2}}, \quad 2 \alpha \notin \mathbb{Z} .
\end{aligned}
$$

Similarly,

$$
\begin{array}{ll}
\left(\Phi^{-1} \Phi_{\lambda}^{\prime}\right)_{22}(-x / 2)=\frac{\alpha+\beta}{x}+\left(\widetilde{d}_{1}+\widetilde{d}_{2} x^{2 \alpha}\right)(1+\mathcal{O}(x)), & \widetilde{d}_{1}=-\frac{\alpha+\beta}{2 \alpha}, \\
\widetilde{d}_{2}=-\frac{\alpha+\beta}{1+2 \alpha} \frac{\Gamma(1+\alpha+\beta) \Gamma(1+\alpha-\beta)}{\Gamma(1-\alpha+\beta) \Gamma(1-\alpha-\beta)} \frac{\Gamma(-2 \alpha)}{\Gamma(1+2 \alpha)^{2}}, & 2 \alpha \notin \mathbb{Z} .
\end{array}
$$

Substituting these expressions into (3.45), we obtain as $x \searrow 0$ :

$$
\begin{aligned}
& w(x)=\frac{\alpha^{2}-\beta^{2}}{x}+\frac{\alpha^{2}-\beta^{2}}{2 \alpha} \\
& \times\left\{1-x^{2 \alpha} \frac{\Gamma(1+\alpha+\beta) \Gamma(1+\alpha-\beta)}{\Gamma(1-\alpha+\beta) \Gamma(1-\alpha-\beta)} \frac{\Gamma(1-2 \alpha)}{\Gamma(1+2 \alpha)^{2}} \frac{1}{1+2 \alpha}\right\}(1+\mathcal{O}(x)), \quad 2 \alpha \notin \mathbb{Z} .
\end{aligned}
$$

Similarly, we verify using (4.67), (4.71), (4.72) that as $x \searrow 0$,

$$
w(x)=\frac{\alpha^{2}-\beta^{2}}{x}+\mathcal{O}(1)+\mathcal{O}\left(x^{2 \alpha}\right)+\mathcal{O}\left(x^{2 \alpha} \ln x\right), \quad 2 \alpha \in \mathbb{Z} .
$$




\subsection{Differential system for $\Psi$}

So far we know that there exist $\delta, M>0$ such that the RH problems for $\Psi$ and $\Phi$ are solvable for $x>M$ and for $0<x<\delta$. We will derive differential equations for $\Psi$ with respect to $x$ and $\zeta$. This will lead to the Painlevé $\mathrm{V}$ equation and will help us to find an identity for the function $w$ given by (3.45) in terms of $v$. Here we follow similar lines as in [16, Section 5.4].

From the RH conditions for $\Psi$, it follows that, for any $x$ for which the $\mathrm{RH}$ problem is solvable, the (matrix) function $A(\zeta ; x)=\Psi_{\zeta}(\zeta ; x) \Psi^{-1}(\zeta ; x)$ is a rational function in $\zeta$ with simple poles at 0 and 1 . Indeed, $A$ is meromorphic because $\Psi$ has constant jump matrices, $A$ is bounded at infinity because of (1.38), and has simple poles at 0 and 1 because of (4.1). Similarly, $B(\zeta ; x)=\Psi_{x}(\zeta ; x) \Psi^{-1}(\zeta ; x)$ is a polynomial of degree 1 in $\zeta$. It follows that $\Psi$ satisfies a linear differential system of the form

$$
\begin{aligned}
& \Psi_{\zeta}(\zeta ; x)=\left[A_{\infty}(x)+\frac{A_{0}(x)}{\zeta}+\frac{A_{1}(x)}{\zeta-1}\right] \Psi(\zeta ; x), \\
& \Psi_{x}(\zeta ; x)=\left[B_{1}(x) \zeta+B_{0}(x)\right] \Psi(\zeta ; x) .
\end{aligned}
$$

Substituting the large $\zeta$-expansion (1.38) for $\Psi$ into (4.88) and (4.89), we can express the coefficient matrices $A_{\infty}, A_{0}, A_{1}, B_{0}$, and $B_{1}$ explicitly in terms of the entries of $C_{1}$ and $C_{2}$ :

$$
\begin{aligned}
& A_{\infty}=-\frac{x}{2} \sigma_{3}, \\
& A_{0}=\left(\begin{array}{cc}
-\beta+q+x r t & -2 \beta r-x h+x r q+r+x r \\
2 \beta t+x j-x t q+t-x t & \beta-q-x r t
\end{array}\right), \\
& A_{1}=\left(\begin{array}{cc}
-q-x r t & 2 \beta r+x h-x r q-r \\
-2 \beta t-x j+x t q-t & q+x r t
\end{array}\right), \\
& B_{1}=-\frac{1}{2} \sigma_{3}, \\
& B_{0}=\left(\begin{array}{cc}
0 & r \\
-t & 0
\end{array}\right),
\end{aligned}
$$

where $q=q(x), r=r(x), t=t(x), h=h(x)$, and $j=j(x)$ are given by

$$
C_{1}(x)=\left(\begin{array}{cc}
q(x) & r(x) \\
t(x) & -q(x)
\end{array}\right), \quad C_{2}(x)=\left(\begin{array}{cc}
* & h(x) \\
j(x) & *
\end{array}\right)
$$

(note that the trace of $C_{1}$ must be zero since the determinant of $\Psi$ is equal to 1 ). Equating the $\mathcal{O}(1 / \zeta)$-terms in $\Psi_{x}=\left(B_{1} \zeta+B_{0}\right) \Psi$ gives the identities

$$
\begin{aligned}
& q^{\prime}(x)=r(x) t(x), \\
& h(x)=-r^{\prime}(x)+r(x) q(x), \\
& j(x)=t^{\prime}(x)+t(x) q(x) .
\end{aligned}
$$

Furthermore, equating the mixed derivatives $\Psi_{x \zeta}=\Psi_{\zeta x}$ leads to the compatibility condition

$$
A_{x}-B_{\zeta}+[A, B]=0, \quad[A, B]=A B-B A .
$$


Let us follow [18, 16] and write

$$
\begin{aligned}
& v(x)=\frac{\alpha+\beta}{2}-q(x)-x q^{\prime}(x)=\frac{\alpha+\beta}{2}-q(x)-x r(x) t(x), \\
& y(x)=\frac{v(x)}{(-2 \beta-1) t(x)-x t^{\prime}(x)}, \\
& u(x)=1+\frac{x t}{(2 \beta+1-x) t(x)+x t^{\prime}(x)} .
\end{aligned}
$$

Using Proposition 4.1 one shows as in [16] that $\operatorname{det} A_{0}=-\frac{(\alpha-\beta)^{2}}{4}$ and $\operatorname{det} A_{1}=-\frac{(\alpha+\beta)^{2}}{4}$. It then follows that the matrices $A_{0}, A_{1}$, and $B_{0}$ can be written in the form (the elements (11), (22), and (21) of $A_{0}$ and $A_{1}$ are easy to verify directly, and (12) follows from the expression for the determinant):

$$
\begin{aligned}
& A_{0}=\left(\begin{array}{cc}
-v+\frac{\alpha-\beta}{2} & u y(v-\alpha+\beta) \\
-\frac{v}{u y} & v-\frac{\alpha-\beta}{2}
\end{array}\right), \\
& A_{1}=\left(\begin{array}{cc}
v-\frac{\alpha+\beta}{2} & -y(v-\alpha-\beta) \\
\frac{v}{y} & -v+\frac{\alpha+\beta}{2}
\end{array}\right), \\
& B_{0}=\frac{1}{x}\left(\begin{array}{cc}
0 & -y[v-\alpha-\beta-u(v-\alpha+\beta)] \\
\frac{1}{y}\left[v-\frac{v}{u}\right] & 0
\end{array}\right) .
\end{aligned}
$$

Writing the compatibility condition (4.99) in terms of the functions $u, v, y$, one verifies that $u, v$, and $y$ solve the system of ODEs

$$
\begin{aligned}
& x u_{x}=x u-2 v(u-1)^{2}+(u-1)[(\alpha-\beta) u-\beta-\alpha], \\
& x v_{x}=u v[v-\alpha+\beta]-\frac{v}{u}(v-\beta-\alpha), \\
& x y_{x}=y\left\{-2 v+\alpha+\beta+u[v-\alpha+\beta]+\frac{v}{u}-x\right\},
\end{aligned}
$$

which is part of the content of Theorem 1.8 (iii). Eliminating $v$ from the first two equations, one shows that $u$ solves the Painlevé V equation (1.22)-(1.23).

Define

$$
\sigma(x)=x q(x)-\frac{\alpha+\beta}{2} x .
$$

It follows from (4.100) that

$$
\sigma^{\prime}=-v
$$

and therefore, by (4.107),

$$
-x \sigma^{\prime \prime}=u v(v-\alpha+\beta)-\frac{v}{u}(v-\beta-\alpha) .
$$

Moreover, in view of (4.96), we have that

$$
\sigma-x \sigma^{\prime}=-x^{2} q^{\prime}=-x^{2} r t \equiv x^{2}\left(B_{0}\right)_{12}\left(B_{0}\right)_{21} .
$$

This equation can be rewritten with the help of (4.105) as

$$
\begin{aligned}
\sigma-x \sigma^{\prime}=-(v-\alpha-\beta- & u(v-\alpha+\beta))\left(v-\frac{v}{u}\right) \\
= & u v(v-\alpha+\beta)+\frac{v}{u}(v-\beta-\alpha)-2 v^{2}+2 \alpha v .
\end{aligned}
$$


Using (4.110), (4.111), and (4.112), we can check directly that the function $\sigma(x)$ satisfies the $\sigma$-form of the fifth Painlevé equation (1.21).

The system (4.88)-(4.89) is the Lax pair associated with Painlevé V. Since the RH problem for $\Psi(\zeta ; x, \alpha, \beta)$ is solvable for $0<x<\delta$ and for $x>M$, the Lax matrices $A_{0}(x ; \alpha, \beta), A_{1}(x ; \alpha, \beta)$, and $B_{0}(x ; \alpha, \beta)$ exist for those values of $x$. However, the system (4.106)-(4.108) has solutions which are meromorphic in $\mathbb{C} \backslash\{0\}$ with a cut from zero to infinity, which implies that $A_{0}, A_{1}$, and $B_{0}$ exist for all but (possibly) a finite number of positive $x$-values. Using appropriately normalized solutions to (4.88)-(4.89), the RH solution $\Psi$ can also be constructed for all but possibly a finite number of positive $x$ values [16]. This proves Theorem 1.8(i) and the equivalent statement for $\Phi$, Proposition 3.1 (i). Furthermore, the differentiability of $\Psi$ with respect to $x$, see (4.89), implies that the asymptotic condition (1.38), and thus also (4.38), holds uniformly as long as $\delta \leq x \leq M$ if $x$ remains bounded away from the set of $x$-values for which the RH problem is not solvable. Together with (4.12) and (4.78), this proves Proposition 3.1 (iii).

Remark 4.2 The functions $u, v, y$ appearing in (4.103)-(4.105) are particular solutions to the system (4.106)-(4.108). Other solutions can be obtained by considering $\mathrm{RH}$ problems for $\Psi$ with modified jump matrices and modified behavior near 0 and 1 , corresponding to different monodromy data, see [2].

Remark 4.3 The $\mathrm{RH}$ problem for $\Psi$ is not the standard $\mathrm{RH}$ problem related to the fifth Painlevé equation. In [16, 18, a RH problem was posed on a contour $U_{0} \cup U_{1} \cup \widehat{\Gamma}$, where $U_{0}$ and $U_{1}$ are small circles surrounding 0 , and 1 , and where $\widehat{\Gamma}=\mathbb{R} \backslash\left(\overline{U_{0}} \cup \overline{U_{1}}\right)$. The equivalence of a particular case of this $\mathrm{RH}$ problem with ours can be verified directly using Proposition 4.1. In order to avoid confusion with the notations in [16], we note that the system (4.106)-(4.108) is written with parameters $\theta_{0}, \theta_{1}$, and $\theta_{\infty}$ in [16], which in our setting are given by

$$
\theta_{0}=-\beta-\alpha, \quad \theta_{1}=\alpha-\beta, \quad \theta_{\infty}=2 \beta .
$$

\section{Proposition 4.4 Set}

$$
a(\zeta ; x)=\left(\Psi(\zeta ; x) \sigma_{3} \Psi^{-1}(\zeta ; x)\right)_{11} .
$$

Then the identities

$$
\begin{aligned}
& \frac{\alpha-\beta}{2} a(0 ; x)=A_{0,11}=-v(x)+\frac{\alpha-\beta}{2}, \\
& \frac{\alpha+\beta}{2} a(1 ; x)=-A_{1,11}=-v(x)+\frac{\alpha+\beta}{2},
\end{aligned}
$$

hold, with $v$ defined as before by (1.41).

Proof. Substituting $\Psi$ expressed from (4.1) into the differential equation

$$
\Psi_{\zeta} \Psi^{-1}=A_{\infty}+\frac{A_{0}}{\zeta}+\frac{A_{1}}{\zeta-1},
$$

and comparing the residue of the left- and right-hand side at 0 leads to an expression for $\Psi(\zeta) \sigma_{3} \Psi(\zeta)^{-1}$ as $\zeta \rightarrow 0$, in terms of $A_{0}$. By (4.103), this gives the first identity. Comparing the residues at 1 gives the second identity. 
Proposition 4.5 Let $w$ be defined by 3.45). Then

$$
\begin{aligned}
& v(x)=-(x w(x))^{\prime}, \\
& \sigma(x)=x w(x), \\
& \sigma(x)=\int_{x}^{+\infty} v(\xi) d \xi .
\end{aligned}
$$

Proof. It follows from Proposition 4.1 that $\Psi$ can be written in the form

$$
\Psi(\zeta)=E(\zeta) \zeta^{\frac{\alpha-\beta}{2} \sigma_{3}}, \quad \Psi(\zeta)=F(\zeta)(\zeta-1)^{-\frac{\alpha+\beta}{2} \sigma_{3}},
$$

with $E$ analytic near 0 and $F$ analytic near 1 . Let us write

$$
\begin{array}{ll}
E(\zeta)=E_{0}\left(I+E_{1} \zeta+\mathcal{O}\left(\zeta^{2}\right)\right), & \text { as } \zeta \rightarrow 0, \\
F(\zeta)=F_{0}\left(I+F_{1}(\zeta-1)+\mathcal{O}\left((\zeta-1)^{2}\right)\right), & \text { as } \zeta \rightarrow 1 .
\end{array}
$$

Substituting (4.120) and (4.121)-(4.122) into (4.89), we obtain the identities

$$
\begin{array}{ll}
E_{0, x}^{\prime}=B_{0} E_{0}, & E_{1, x}^{\prime}=E_{0}^{-1} B_{1} E_{0}, \\
F_{0, x}^{\prime}=B_{0} F_{0}, & F_{1, x}^{\prime}=F_{0}^{-1} B_{1} F_{0},
\end{array}
$$

which imply by Proposition 4.4, in particular, that

$$
E_{1,22}^{\prime}(x)=\left(E_{0}^{-1} B_{1} E_{0}\right)_{22}=\left(E_{0} B_{1} E_{0}^{-1}\right)_{22}=\frac{1}{2} a(0 ; x), \quad F_{1,11}^{\prime}(x)=-\frac{1}{2} a(1 ; x) .
$$

On the other hand recalling equation (4.83), we obtain

$$
\begin{aligned}
& \left(\Phi^{-1}\left(-\frac{x}{2} ; x\right) \Phi_{\lambda}^{\prime}\left(-\frac{x}{2} ; x\right)\right)_{22}=-\frac{1}{2}+\frac{\alpha+\beta}{2 x}+\frac{1}{x} E_{1,22}, \\
& \left(\Phi^{-1}\left(\frac{x}{2} ; x\right) \Phi_{\lambda}^{\prime}\left(\frac{x}{2} ; x\right)\right)_{11}=\frac{1}{2}-\frac{\alpha-\beta}{2 x}+\frac{1}{x} F_{1,11} .
\end{aligned}
$$

From (3.45), it follows that

$$
w(x)=-\frac{\alpha}{2}+\frac{\alpha^{2}-\beta^{2}}{2 x}+\frac{\alpha-\beta}{2 x} E_{1,22}-\frac{\alpha+\beta}{2 x} F_{1,11},
$$

and by (4.123) together with Proposition 4.4 we obtain $-(x w(x))^{\prime}=v(x)$. From (4.110) and (4.117), it follows that $\sigma(x)=x w(x)+$ constant, where $\sigma$ is defined by (4.109). To determine the constant, note first that, as follows from (4.14) and (4.17), $q(x)=C_{1,11} \rightarrow(\alpha+\beta) / 2$ as $x \rightarrow+\infty$, and hence, $\sigma(x) \rightarrow 0$ as $x \rightarrow+\infty$. On the other hand, as follows from (4.10), (4.11), we have $x w(x) \rightarrow 0$ as $x \rightarrow+\infty$. Hence the constant in question is zero, and we obtain (4.118). Equation (4.119) is obtained similarly.

Combining (4.117), (4.86), (4.87), and (4.22), we obtain (1.46). The expressions (4.86), (4.87), (4.118), and (4.119) imply (1.47).

Proposition 4.6 Let $v$ be defined by 1.41). Then $v(x)$ is real for $x>0$ if $\operatorname{Im} \alpha=0$, $\alpha>-\frac{1}{2}$, and $\operatorname{Re} \beta=0$. 
Proof. Suppose that $\alpha>-\frac{1}{2}, \operatorname{Re} \beta=0$, and that $\Psi(\zeta ; x)$ is a solution to the $\mathrm{RH}$ problem for $\Psi$ given in Section 1.3. Then it is straightforward to verify that the function $\widehat{\Psi}$ defined by

$$
\widehat{\Psi}(\zeta):=\sigma_{1} \overline{\Psi(-\overline{(\zeta-1 / 2)})} \sigma_{1} e^{ \pm \pi i \beta \sigma_{3}}, \quad \text { if } \pm \operatorname{Im} \zeta>0
$$

with $\sigma_{1}=\left(\begin{array}{ll}0 & 1 \\ 1 & 0\end{array}\right)$, solves the RH problem for $\Psi(\zeta+1 / 2)$ for real $x$ up to a constant factor. Therefore, by uniqueness,

$$
\Psi(\zeta+1 / 2)=C(x)^{\sigma_{3}} \widehat{\Psi}(\zeta)
$$

where $C(x)$ is independent of $\zeta$. By (4.114), it follows that $a(0 ; x)=\overline{a(1 ; x)}$. Subtracting the complex conjugate of (4.116) from (4.115), we conclude that $v(x)=\overline{v(x)}$.

\subsection{Solvability of the $\mathrm{RH}$ problem for $\Psi$}

In this section, we will prove Theorem 1.8 (ii) and Proposition 3.1 (ii): we will prove that the RH problem for $\Psi$ is solvable for all positive values of $x$ if $\operatorname{Re} \beta=0$ and $\operatorname{Im} \alpha=0, \alpha>-\frac{1}{2}$.

\subsubsection{Vanishing lemma for Painlevé V}

For a general class of $\mathrm{RH}$ problems, it is known that solvability of a $\mathrm{RH}$ problem is equivalent to the triviality of a homogeneous version of the RH problem [16, 19, 28]. For the case of Painlevé V, this has been used in [18] for a slightly different but equivalent $\mathrm{RH}$ problem (cf. Remark4.3). In our case a sufficient (and necessary) condition to prove the solvability of the $\mathrm{RH}$ problem for $\Psi$ is given by the following so-called vanishing lemma.

Lemma 4.7 (Vanishing lemma for Painlevé V) Let $x>0, \operatorname{Im} \alpha=0, \operatorname{Re} \beta=0$, and suppose that $\Psi_{0}$ satisfies the $R H$ conditions (a), (b), (d0), and (d1) of the RH problem for $\Psi$, with condition (c) replaced by the homogeneous asymptotic condition

$$
\Psi_{0}(\zeta) e^{\frac{x}{2} \zeta \sigma_{3}}=\mathcal{O}\left(\zeta^{-1}\right), \quad \text { as } \zeta \rightarrow \infty
$$

Then it follows that $\Psi_{0} \equiv 0$.

Remark 4.8 A vanishing lemma was proven in [18] for a family of solutions to the system (4.106)-(4.108). Our solution, however, is not contained in this family, and the vanishing lemma requires a different proof in our case. For the proof of the vanishing lemma, we follow similar lines as in [12, Section 5.3].

Proof of Lemma 4.7. Suppose we have a solution $\Psi_{0}$ to the homogeneous $\mathrm{RH}$ problem. We will then prove that $\Psi_{0} \equiv 0$. Let us first define a function $M$ as follows,

$$
\begin{array}{ll}
M(\zeta)=\Psi_{0}\left(\zeta+\frac{1}{2}\right) e^{\frac{x}{2} \zeta \sigma_{3}}, & \text { if } \frac{3 \pi}{4}<\arg \zeta<\frac{5 \pi}{4} \\
M(\zeta)=\Psi_{0}\left(\zeta+\frac{1}{2}\right) e^{-\pi i \beta \sigma_{3}} e^{\frac{x}{2} \zeta \sigma_{3}}, & \text { for } 0<\arg \zeta<\frac{\pi}{4} \\
M(\zeta)=\Psi_{0}\left(\zeta+\frac{1}{2}\right) e^{\pi i \beta \sigma_{3}} e^{\frac{x}{2} \zeta \sigma_{3}}, & \text { for } \frac{7 \pi}{4}<\arg \zeta<2 \pi
\end{array}
$$


In the remaining regions where $\frac{\pi}{4}<\arg \zeta<\frac{3 \pi}{4}$ or $\frac{5 \pi}{4}<\arg \zeta<\frac{7 \pi}{4}$ we define $M$ as the analytic continuation of $M$ from the other sectors in such a way that $M$ has jumps only on the imaginary and on a part of the real axis:

$$
\begin{array}{ll}
M(\zeta)=\Psi_{0}\left(\zeta+\frac{1}{2}\right)\left(\begin{array}{cc}
1 & -e^{\pi i(\alpha-\beta)} \\
0 & 1
\end{array}\right) e^{-\pi i \beta \sigma_{3}} e^{\frac{x}{2} \zeta \sigma_{3}}, & \text { for } \frac{\pi}{4}<\arg \zeta<\frac{\pi}{2}, \\
M(\zeta)=\Psi_{0}\left(\zeta+\frac{1}{2}\right)\left(\begin{array}{cc}
1 & 0 \\
-e^{-\pi i(\alpha-\beta)} & 1
\end{array}\right) e^{\frac{x}{2} \zeta \sigma_{3}}, & \text { for } \frac{\pi}{2}<\arg \zeta<\frac{3 \pi}{4} \\
M(\zeta)=\Psi_{0}\left(\zeta+\frac{1}{2}\right)\left(\begin{array}{cc}
1 & 0 \\
-e^{\pi i(\alpha-\beta)} & 1
\end{array}\right) e^{\frac{x}{2} \zeta \sigma_{3}}, & \text { for } \frac{5 \pi}{4}<\arg \zeta<\frac{3 \pi}{2} \\
M(\zeta)=\Psi_{0}\left(\zeta+\frac{1}{2}\right)\left(\begin{array}{cc}
1 & -e^{-\pi i(\alpha-\beta)} \\
0 & 1
\end{array}\right) e^{\pi i \beta \sigma_{3}} e^{\frac{x}{2} \zeta \sigma_{3}}, & \text { for } \frac{3 \pi}{2}<\arg \zeta<\frac{7 \pi}{4}
\end{array}
$$

Then $M$ satisfies the following $\mathrm{RH}$ conditions.

\section{RH problem for $M$}

(a) $M$ is analytic in $\mathbb{C} \backslash\left(i \mathbb{R} \cup\left[-\frac{1}{2}, \frac{1}{2}\right]\right)$.

(b) $M$ satisfies the following jump conditions on the contour $\left(i \mathbb{R} \cup\left(-\frac{1}{2}, \frac{1}{2}\right)\right)$, with $i \mathbb{R}$ oriented upwards and $\left(-\frac{1}{2}, \frac{1}{2}\right)$ oriented from left to right,

$$
\begin{array}{ll}
M_{+}(\zeta)=M_{-}(\zeta) V_{1}(\zeta), & \text { as } \zeta \in(0,+i \infty), \\
M_{+}(\zeta)=M_{-}(\zeta) V_{2}(\zeta), & \text { as } \zeta \in(-i \infty, 0), \\
M_{+}(\zeta)=M_{-}(\zeta) e^{-\pi i(\alpha-\beta) \sigma_{3}}, & \text { as } \zeta \in\left(-\frac{1}{2}, 0\right), \\
M_{+}(\zeta)=M_{-}(\zeta) e^{-\pi i(\alpha+\beta) \sigma_{3}}, & \text { as } \zeta \in\left(0, \frac{1}{2}\right),
\end{array}
$$

with

$$
\begin{aligned}
& V_{1}(\zeta)=\left(\begin{array}{cc}
0 & e^{\pi i \alpha} e^{-x \zeta} \\
-e^{-\pi i \alpha} e^{x \zeta} & e^{-\pi i \beta}
\end{array}\right), \\
& V_{2}(\zeta)=\left(\begin{array}{cc}
0 & e^{-\pi i \alpha} e^{-x \zeta} \\
-e^{\pi i \alpha} e^{x \zeta} & e^{\pi i \beta}
\end{array}\right),
\end{aligned}
$$

(c) For fixed $x>0$,

$$
M(\zeta)=\mathcal{O}\left(\zeta^{-1}\right), \quad \text { as } \zeta \rightarrow \infty .
$$

(d0) As $\zeta \rightarrow-\frac{1}{2}$,

$$
M(\zeta)=\mathcal{O}\left(\begin{array}{ll}
\left|\zeta+\frac{1}{2}\right|^{\frac{\alpha}{2}} & \left|\zeta+\frac{1}{2}\right|^{-\frac{\alpha}{2}} \\
\left|\zeta+\frac{1}{2}\right|^{\frac{\alpha}{2}} & \left|\zeta+\frac{1}{2}\right|^{-\frac{\alpha}{2}}
\end{array}\right)
$$

(d1) As $\zeta \rightarrow+\frac{1}{2}$,

$$
M(\zeta)=\mathcal{O}\left(\begin{array}{ll}
\left|\zeta-\frac{1}{2}\right|^{-\frac{\alpha}{2}} & \left|\zeta-\frac{1}{2}\right|^{\frac{\alpha}{2}} \\
\left|\zeta-\frac{1}{2}\right|^{-\frac{\alpha}{2}} & \left|\zeta-\frac{1}{2}\right|^{\frac{\alpha}{2}}
\end{array}\right)
$$


Let us now define a function $H(\zeta)$ in terms of $M$ and its Hermitian conjugate as follows:

$$
H(\zeta)=M(\zeta) M^{*}(-\bar{\zeta})
$$

Because of the condition (c) of the RH problem for $M$, we have that $H(\zeta)=\mathcal{O}\left(\zeta^{-2}\right)$ as $\zeta \rightarrow \infty$. Furthermore, using the jump condition (4.131) (4.132) for $M$, we obtain that $H$ has no jump across $\left(0, \frac{1}{2}\right)$. (This is only true if $\operatorname{Re} \beta=0, \operatorname{Im} \alpha=0$.) Therefore, $H$ is meromorphic for $\operatorname{Re} \zeta>0$, with an isolated singularity at $\frac{1}{2}$, which is removable because of (4.136) and (4.137). Using Cauchy's theorem, we then have

$$
\int_{-i \infty}^{+i \infty} H_{-}(\zeta) d \zeta=0, \quad \int_{-i \infty}^{+i \infty} H_{-}^{*}(\zeta) d \zeta=0
$$

Because of the jump conditions for $M$, the first integral implies that

$$
\int_{-i \infty}^{0} M_{-}(\zeta) V_{2}^{*}(\zeta) M_{-}^{*}(\zeta) d \zeta+\int_{0}^{+i \infty} M_{-}(\zeta) V_{1}^{*}(\zeta) M_{-}^{*}(\zeta) d \zeta=0
$$

Summing up this expression and the one obtained from the second integral in (4.139), we find, using (4.133), (4.134) and the fact that $x$ is real,

$$
\int_{-i \infty}^{0} M_{-}(\zeta)\left(\begin{array}{cc}
0 & 0 \\
0 & 2 e^{\pi i \beta}
\end{array}\right) M_{-}^{*}(\zeta) d \zeta+\int_{0}^{i \infty} M_{-}(\zeta)\left(\begin{array}{cc}
0 & 0 \\
0 & 2 e^{-\pi i \beta}
\end{array}\right) M_{-}^{*}(\zeta) d \zeta=0 .
$$

Since $\operatorname{Re} \beta=0$, it follows immediately that the second column of $M_{-}$is identically zero on $i \mathbb{R} \backslash\{0\}$. From the jump conditions (4.129)-(4.130), it then follows that the first column of $M_{+}$is zero on $i \mathbb{R} \backslash\{0\}$ as well. Therefore, we have that $M_{j 2}(\zeta)=0$ for $\operatorname{Re} \zeta>0$, and $M_{j 1}(\zeta)=0$ for $\operatorname{Re} \zeta<0$. Let us now define

$$
g_{j}(\zeta)= \begin{cases}M_{j 2}(\zeta), & \text { as } \operatorname{Re} \zeta<0, \\ M_{j 1}(\zeta), & \text { as } \operatorname{Re} \zeta>0,\end{cases}
$$

so that $g_{j}$ is analytic in $\mathbb{C} \backslash\left(i \mathbb{R} \cup\left[-\frac{1}{2}, \frac{1}{2}\right]\right)$. Furthermore, $g_{j}$ is bounded except near $\pm \frac{1}{2}$. On $i \mathbb{R}, g$ has the following jump relation,

$$
g_{j,+}(\zeta)=g_{j,-}(\zeta) \times \begin{cases}e^{\pi i \alpha} e^{-x \zeta}, & \text { as } \zeta \in(0,+i \infty), \\ e^{-\pi i \alpha} e^{-x \zeta}, & \text { as } \zeta \in(-i \infty, 0)\end{cases}
$$

Now we write $\widehat{g}$ for the analytic continuation of $g$ from the left half plane to $\mathbb{C} \backslash\left[-\frac{1}{2},+\infty\right)$,

$$
\widehat{g}(\zeta)= \begin{cases}g(\zeta), & \text { as } \operatorname{Re} \zeta<0, \\ g(\zeta) e^{\pi i \alpha} e^{-x \zeta}, & \text { as } \operatorname{Re} \zeta>0, \operatorname{Im} \zeta>0, \\ g(\zeta) e^{-\pi i \alpha} e^{-x \zeta}, & \text { as } \operatorname{Re} \zeta>0, \operatorname{Im} \zeta<0\end{cases}
$$

Set

$$
h(\zeta)=\widehat{g}\left(-(\zeta+1)^{3 / 2}\right)
$$

It is now easy to verify that $h$ is analytic and bounded for $\operatorname{Re} \zeta \geq 0$, and that $h(\zeta)=$ $\mathcal{O}\left(e^{-x|\zeta|}\right)$ for $\zeta \rightarrow \pm i \infty$. By Carlson's theorem, this implies that $h \equiv 0$ if $x>0$. Tracing back the previous steps, it follows that $g \equiv 0, M \equiv 0$, and $\Psi_{0} \equiv 0$, which proves the vanishing lemma. 
Remark 4.9 The proof of the vanishing lemma does not apply if either $\alpha$ is not real or $\beta$ is not purely imaginary. The first failure is that the function $H$ would not be analytic across $\left(0, \frac{1}{2}\right)$ in this case. A further problem in the proof would be that the matrices $V_{1}+V_{1}^{*}$ and $V_{2}+V_{2}^{*}$ lose their symmetry, which results in non-zero off-diagonal entries in (4.141). It is of course possible that the vanishing lemma can be proven in a different way. Another possibility is that, given $\alpha$ and $\beta$, the $\mathrm{RH}$ problem is not solvable for certain isolated values of $x$.

\section{Asymptotics for Toeplitz determinants}

Using the identities of Proposition 4.4 and the Fourier representation for $V(z)$, we can rewrite (3.46) in the form, with $x=2 n t$,

$$
\begin{aligned}
& \frac{d}{d t} \ln D_{n}=(\alpha+\beta) n-\left(\alpha^{2}-\beta^{2}\right) \frac{e^{-t}}{\sinh t}+(\alpha-\beta) \sum_{k=1}^{\infty} k V_{k} e^{-k t}+(\alpha+\beta) \sum_{k=1}^{\infty} k V_{-k} e^{-k t} \\
& +\frac{1}{t} \sigma(x)-v(x)\left\{\alpha+\alpha\left(\frac{1}{t}-\frac{e^{-t}}{\sinh t}\right)+2 \sum_{k=1}^{\infty} k\left(V_{k}+V_{-k}\right) \cosh (k t)\right\}+O(1 / n) \widetilde{\Phi}(x) .
\end{aligned}
$$

The expressions (4.86), (4.87), (4.118) yield the $x \rightarrow 0$ expansion for $\sigma$ in (1.26); and the expressions (4.22), (4.119) imply the $x \rightarrow+\infty$ expansion in (1.26).

Because of the uniformity property of the error term in (5.1), the integration of this identity from $\varepsilon>0$ to some $t<t_{0}$ gives uniformly for any $0<\varepsilon<t$,

$$
\begin{aligned}
& \ln D_{n}(t)=\ln D_{n}(\varepsilon)+(\alpha+\beta) n(t-\varepsilon)+\sum_{k=1}^{\infty} k\left[V_{k}-(\alpha+\beta) \frac{e^{-t k}}{k}\right]\left[V_{-k}-(\alpha-\beta) \frac{e^{-t k}}{k}\right] \\
& -\sum_{k=1}^{\infty} k V_{k} V_{-k}+(\alpha-\beta) \sum_{k=1}^{\infty} V_{k} e^{-k \varepsilon}+(\alpha+\beta) \sum_{k=1}^{\infty} V_{-k} e^{-k \varepsilon} \\
& +\left[\int_{2 n \varepsilon}^{2 n t} \frac{\sigma(x)}{x} d x+\left(\alpha^{2}-\beta^{2}\right) \ln \left\{n\left(1-e^{-2 \varepsilon}\right)\right\}\right]-\left(\alpha^{2}-\beta^{2}\right) \ln n+R_{n}(t)+\mathcal{O}(1 / n) ; \\
& R_{n}(t)=-\int_{\varepsilon}^{t} v(2 n t)\left\{\alpha+\alpha\left(\frac{1}{t}-\frac{e^{-t}}{\sinh t}\right)+2 \sum_{k=1}^{\infty} k\left(V_{k}+V_{-k}\right) \cosh (k t)\right\} d t .
\end{aligned}
$$

If $\alpha$ is real and $\beta$ is imaginary, we can take as a path of integration the interval $[\varepsilon, t]$ of the real line as, according to Section 4.4, the functions $\sigma(x)$ and $v(x)$ are real analytic for positive $x$. The estimates (1.26), (1.46) ensure integrability at $x=0$ and $x=+\infty$. In particular, the term in the square brackets in (5.2) converges if $\varepsilon \rightarrow 0$.

For arbitrary $\beta, \operatorname{Re} \alpha>-1 / 2$, we can choose a path of integration and the endpoint $t$ to avoid possible singular points $\left\{x_{1}, \ldots, x_{k}\right\}$. The estimates (1.26), (1.46) were obtained above for positive $x$. The restriction to real $x$ was only imposed for simplicity of notation. In fact, it is easy to verify that the estimates (1.26), (1.46) hold for any path to zero and infinity within a sector $-\pi / 2+\delta<\arg x<\pi / 2+\delta, 0<\delta<\pi / 2$.

We have for $R_{n}(t)$ in (5.2):

$$
\left|R_{n}(t)\right|<C \int_{0}^{t}|v(2 n u)| d u=\mathcal{O}(1 / n), \quad n \rightarrow \infty, \quad 0<t<t_{0} .
$$


Now recall that (5.2) is uniform in $\varepsilon$, and $\ln D_{n}(t)$ is continuous at $t=0$. Therefore, taking the limit $\varepsilon \rightarrow 0$ in (5.2) and using the Fisher-Hartwig asymptotics (1.5) for $\ln D_{n}(0)$ gives the expression (1.24) of Theorem 1.1 .

This concludes the proof of both Theorem 1.1 and Theorem 1.4 .

\section{Acknowledgements}

The authors are grateful to Alexander Abanov, Yan Fyodorov, and Jon Keating for encouraging our interest in transition asymptotics for determinants. Tom Claeys is a Postdoctoral Fellow of the Fund for Scientific Research - Flanders (Belgium), and was also supported by the ESF program MISGAM. Alexander Its was supported in part by NSF grant \#DMS-0701768. Igor Krasovsky was supported in part by EPSRC grant \#EP/E022928/1.

\section{References}

[1] F. V. Andreev, On special solutions of the fifth Painlevé equation, J. Math. Sci. 99 (2000), no. 1, 802-807.

[2] F.V. Andreev and F. V. Kitaev, On connection formulas for the asymptotics of some special solutions of the fifth Painlevé equation. J. Math. Sci. 99 (2000), no. $1,808-815$.

[3] F.V. Andreev and A.V. Kitaev, Connection formulae for asymptotics of the fifth Painlevé transcendent on the real axis. Nonlinearity 13 (2000), no. 5, 1801-1840.

[4] J. Baik, P. Deift, and K. Johansson, On the distribution of the length of the longest increasing subsequence of random permutations, J. Amer. Math. Soc. 12 (1999), $1119-1178$.

[5] Bateman, Erdelyi. Higher transcendental functions, New York: McGraw-Hill, $1953-1955$

[6] E. Basor, Asymptotic formulas for Toeplitz determinants, Trans. Amer. Math. Soc. 239 (1978), 33-65.

[7] E. L. Basor and C. A. Tracy, Asymptotics of a tau-function and Toeplitz determinants with singular generating functions, International J. of Mod. Phys. A 7, Suppl. 1A (1992), 83-107

[8] A. Böttcher, B. Silbermann, Toeplitz operators and determinants generated by symbols with one Fisher-Hartwig singularity. Math. Nachr. 127 (1986), 95-123

[9] A. Böttcher, B. Silbermann, Toeplitz matrices and determinants with FisherHartwig symbols. J. Funct. Anal. 63 (1985), 178-214

[10] A. Böttcher, H. Widom, Two elementary derivations of the pure Fisher-Hartwig determinant. Int. Eq. Op. Th. 53 (2005), 593-596

[11] P. Deift, A. Its, and I. Krasovsky, Toeplitz and Hankel determinants with FisherHartwig singularities arXiv:0905.0443. 
[12] P. Deift, T. Kriecherbauer, K.T-R McLaughlin, S. Venakides, and X. Zhou, Uniform asymptotics for polynomials orthogonal with respect to varying exponential weights and applications to universality questions in random matrix theory, Comm. Pure Appl. Math. 52 (1999), 1335-1425.

[13] P. Deift, T. Kriecherbauer, K.T-R McLaughlin, S. Venakides, and X. Zhou, Strong asymptotics of orthogonal polynomials with respect to exponential weights, Comm. Pure Appl. Math. 52 (1999), 1491-1552.

[14] P. Deift and X. Zhou, A steepest descent method for oscillatory Riemann-Hilbert problems. Asymptotics for the MKdV equation, Ann. Math. 137 (1993), no. 2, $295-368$.

[15] T. Ehrhardt, A status report on the asymptotic behavior of Toeplitz determinants with Fisher-Hartwig singularities, Operator Theory: Adv. Appl. 124, 217241 (2001).

[16] A.S. Fokas, A.R. Its, A.A. Kapaev, and V.Yu. Novokshenov, "Painlevé transcendents: the Riemann-Hilbert approach", AMS Mathematical Surveys and Monographs 128 (2006).

[17] A.S. Fokas, A.R. Its, and A.V. Kitaev, The isomonodromy approach to matrix models in 2D quantum gravity, Comm. Math. Phys. 147 (1992), 395-430.

[18] A.S. Fokas, U. Mugan, and X. Zhou, On the solvability of Painlevé I, III and V, Inverse Problems 8 (1992), no. 5, 757-785.

[19] A.S. Fokas and X. Zhou, On the solvability of Painlevé II and IV, Comm. Math. Phys. 144 (1992), no. 3, 601-622.

[20] F. Franchini and A.G. Abanov, Asymptotics of Toeplitz determinants and the emptiness formation probability for the XY spin chain, J. Phys. A: Math. Gen. 38 (2005), 5069-5095.

[21] B. L. Golinskii and I. A. Ibragimov, A limit theorm of G. Szegő. (Russian) Izv. Akad. Nauk SSSR Ser. Mat. 35 (1971), 408-427.

[22] I. A. Ibragimov, A theorem of Gabor Szegö. (Russian) Mat. Zametki 3 (1968) 693-702.

[23] A. Its and I. Krasovsky, Hankel determinant and orthogonal polynomials for the Gaussian weight with a jump, Contemp. Math. 458 (2008), 215-247.

[24] A. Its, C. Tracy, H. Widom, Random words, Toeplitz determinants and integrable systems. II, Phys. D 152/153 (2001), 199-224.

[25] M. Jimbo, Monodromy problem and the boundary condition for some Painlevé equations, Publ. RIMS, Kyoto Univ. 18 (1982), 1137-1161.

[26] M. Jimbo and T. Miwa, Studies on holonomic quantum fields XVII, Proc. Japan Acad. 56 A (1980), $405-410$.

[27] K. Johansson, On Szegős asymptotic formula for Toeplitz determinants and generalizations, Bull. Sci. Math. (2) 112 (1988), no. 3, 257-304. 
[28] S. Kamvissis, K.D.T-R McLaughlin, and P.D. Miller, "Semiclassical soliton ensembles for the focusing nonlinear Schrödinger equation", Ann. Math. Studies 154, Princeton Univ. Press, Princeton (2003).

[29] B. M. McCoy, The connection between statistical mechanics and quantum field theory [arxiv: hep-th/9403084

[30] B. M. McCoy, C. A. Tracy and T. T. Wu, Painlevé functions of the third kind, J. Math. Phys. 18 (1977), 1058-1092

[31] B.M. McCoy and S. Tang, Connection formulae for Painlevé functions. Solitons and coherent structures (Santa Barbara, Calif., 1985), Phys. D 18 (1986), no. 1-3, 190-196.

[32] B. M. McCoy and T. T. Wu, The two-dimensional Ising model. Harvard Univ. Press: Cambridge MA, 1973.

[33] P. Shukla, Level spacing functions and the connection problem of a fifth Painlevé transcendent, J. Phys. A 28 (1995), no. 11, 3177-3195.

[34] C. A. Tracy, Asymptotics of a tau function arising in the two-dimensional Ising model, Commun. Math. Phys. 142 (1991), 297-311.

[35] H. Widom. Toeplitz determinants with singular generating functions. Amer. J. Math. 95 (1973), 333-383

[36] T. T. Wu, B. M. McCoy, C. A. Tracy and E. Barouch, Spin-spin correlation functions for the two-dimensional Ising model: Exact theory in the scaling region, Phys. Rev. B13 (1976), 316-374 